\title{
À Suwałki en Pologne et au Canada. Tziganes, Indiens, métis et êtres hybrides. Du cliché de l'étranger à l'hybridité exilique constitutive de la condition humaine
}

Piotr Bilos

Inalco

Dans Exil et modernité (Bilos, 2012), j'interrogeais la façon dont un exil de nature initialement, semblait-il, géo-politique, et impliquant la rupture physique avec la culture (nationale) des origines, s'associait au phénomène de l'exil moderne tel que le concevait par exemple Emmanuel Kant (renoncement à la patrie de l'enfance au bénéfice de l'édification d'une société qui se voudra rationnelle). Cet exil avait pour corollaire des stratégies d'écriture novatrices (journaux et essais principalement), lesquelles venaient assouplir la dichotomie patrie/société née des Lumières avec le dessein de dépasser celle-ci. Dans cette perspective, le sujet scripteur se voyait imposer un éloignement du «pays» des origines lui donnant la possibilité de se reconstruire au sein de l'espace d'arrivée mais aussi en décalage par rapport à celui-ci.

Cet article, d'une façon plus ciblée, interroge deux ouvrages qui, s'ils adoptent eux aussi l'altérité comme objet d'exploration et opèrent - pour ainsi dire - en situation d'altérité, sont portés par un sujet scripteur qui, apparemment, ne tombe pas lui-même sous le coup de l'exil : libre de ses mouvements, c'est en vertu d'une décision pleinement volontaire qu'il orchestre sa rencontre avec l'altérité. Néanmoins, le décentrement du point de vue, force motrice du projet d'écriture lui-même, s'avère indissociable dans les deux cas d'une fréquentation tous azimuts et prolongée de l'autre, laquelle a pour effet de remettre en question les clichés 
SLOVO

154 Les Voyages lointains des écrivains polonais $\left(\mathrm{XX}^{\mathrm{e}}-\mathrm{XXI}^{\mathrm{e}}\right.$ siècles $)-\mathrm{n}^{\circ} 51$

et les clivages hérités de la culture d'appartenance ou produits tout simplement par l'ignorance. Certes, le sujet scripteur s'immerge dans la vie de communautés étrangères (de son point de vue), en tant qu'observateur appartenant à un cadre de référence externe (du point de vue de ces communautés), mais l'empathie tout autant que le regard critique qu'il porte sur elles, le déportent vers des problématiques telles que le brouillage des frontières et le métissage ainsi que l'hybridité. Ainsi, il est amené à prendre ses distances à l'égard de ce que l'on pourrait nommer une logique binaire essentialiste. Vouloir fréquenter l'autre, chercher à le comprendre tout en gardant les yeux ouverts sur ce qui sera perçu comme des défauts ou des faiblesses, cette aventure ne laisse pas indemne.

Les «voyages » initiatiques (l'un réel et géographique, l'autre davantage mental et né de la fréquentation sur place de communautés allogènes) auquel cet article se réfère, tout en faisant ressortir la valeur opératoire de la frontière entre le chez-soi et l'autre, déstabilisent celle-ci et ouvrent les vannes à une compréhension de la condition humaine en tant qu'exilique dans un sens qui rejoint les réflexions d'Edward Saïd (Saïd, 1996, p. 68-69) :

Métaphysiquement parlant, l'exil est pour l'intellectuel un état d'inquiétude, un mouvement où, constamment déstabilisé, il déstabilise les autres. Pas plus qu'il ne lui est possible de revenir en arrière et de retrouver la stabilité de son «chez-soi », il ne peut davantage, hélas, se reconnaître pleinement dans son nouveau pays »; « aux limites de la pathologie », l'intellectuel fait de son exil « un lieu d'existence à part entière, fût-il provisoire ».

Le premier ouvrage, Chyba za nami nie traficie [Je doute que vous parveniez à nous suivre, Milewski, 2013] est un recueil de quatorze « récits tziganes » de Jacek Milewski ; son auteur, un Polonais, a cofondé et dirigé l'unique école bilingue romani-polonais dans son pays (de 1993 à 2009)․․ Milewski élabore des fictions

1. Comme l'indique Jacek Milewski dans un entretien paru dans un bulletin spécialisé dédié aux Tziganes polonais, cette école se distinguait des nombreuses classes roms existant dans tout le pays, adossées à des établissements scolaires publics standards. Ces dernières ont été supprimées en 2008 par le ministère inquiet des risques de «ghettoïsation ». L'école de Suwałki, elle, constituait une entité parfaitement autonome. Selon Milewski, le problème de la scolarisation des élèves tziganes s'avère particulièrement complexe. Quels que soient les avantages liés à l'assimilation, celle-ci entraîne à terme le risque d'une perte de leur identité culturelle. Milewski, pour sa part, a commencé à fréquenter les Tziganes à partir de 1992, lorsqu'après des études de théologie à Varsovie, il rejoint sa ville natale de Suwałki (ville de l'extrême nord-est de la Pologne actuelle) et découvre, grâce au père Jerzy Zawadzki, 
À Suwałki en Pologne et au Canada. Tziganes, Indiens, métis et êtres hybrides. Du cliché de l'étranger à l'hybridité exilique constitutive de la condition humaine

Piotr Bilos

censées dire la vérité et s'il se réserve le droit de lâcher la bride à son imagination, il n'a de cesse d'exploiter les observations récoltées au cours de sa longue fréquentation des différents milieux tziganes polonais ainsi que sa connaissance intime de cette culture allogène et de son histoire ${ }^{2}$. Ces récits n'obéissent pas à un schéma unique, et l'ensemble revêt une allure résolument polymorphe. Le cycle tzigane milewskien favorise la narration à la troisième personne tout en autorisant l'apparition occasionnelle d'un «je », alter ego de l'auteur dont la fonction principale reste toutefois la mise en valeur les personnages tziganes. Ce qui ressort également est l'oralité truculente des échanges dialogués ${ }^{3}$ et le lyrisme des parties narratives ${ }^{4}$ pouvant s'associer à la poétique des contes de fées. Un autre composant

l'analphabétisme des enfants tziganes, lesquels ne fréquentent pas l'école. C'est alors que naît l'idée d'une école adaptée à leurs besoins. Progressivement, Milewski s'immerge dans ce milieu jusqu’à y découvrir une vocation. Voir MILEWSKI, 2014.

2. Je traduis toutes les citations provenant des ouvrages de Fiedler et Milewski.

3. Milewski a pris le parti d'utiliser le polonais dans les dialogues, mais il émaille ceux-ci de phrases retranscrites à partir du dialecte romani de la communauté Polska Roma, la plus ancienne installée en territoire polonais, la plus attachée aux traditions et conservatrice dans ses comportements. Alors qu'une partie des Tziganes tend à perdre l'usage de leur langue, ceux de la région de Suwałki où Milewski a fondé son école parlent majoritairement le dialecte, lequel reste vivace au sein de cette communauté en général. En réponse à ma question, Milewski affirme que les Tziganes Polska Roma parlent le dialecte mais emploient à l'occasion des termes polonais (bien que déclinés à la façon romani), surtout lorsqu'il s'agit de termes dénotant des réalités nouvelles non prises en charge par le dialecte. Cela signifie que les proportions polonais-dialecte romani telles qu'elles apparaissent dans le livre (au bénéfice du polonais) sont en raison inverse de ce que l'on observe dans la réalité.

4. Dans le récit intitulé «Bonne nuit les enfants » [Dobranocka], lorsqu'un grand-père endosse le rôle du conteur-narrateur et entreprend de raconter « la geste » des Tziganes à ses petits-enfants à l'heure du coucher, son récit se charge de lyrisme. Les phrases adoptent un rythme musical, travaillées par l'influx vital d'un discours visant à suspendre le temps extra-verbal, apaiser les souffrances et, au bout du compte, apprivoiser la mort, puisque après avoir évoqué le voyage en tant qu'expérience de contact privilégié avec le monde, les différentes activités professionnelles des Tziganes (notamment à la cour du roi Jean Sobieski), le récit bifurque vers le « Porajmos », c'est-à-dire l'Holocauste des Tziganes. Le lecteur apprend alors que la scène se passe à Berlin (bien que les protagonistes soient des Tziganes polonais) et s'achève sur une note philosophique et empreinte de merveilleux : alors que les enfants savourent enfin la paix du sommeil, la lune reprend le fil du récit et s'adresse en polonais au grand-père en ces termes: «Tu n'as pas lu Andrzej Bobkowski, mais après toutes ses années, tu devrais savoir que les Allemands nous ont pardonné tout ce qu' ils nous ont infligé ; rien de sert de se fâcher/Nie czytateś Andrzeja Bobkowskiego, ale po tylu latach powinieneś wiedzieć, że Niemcy nam wybaczyli wszystko, co nam zrobili - nie ma co sięgniewaće », MILEWSKI, 2013, p. 144. 
SLOVO

156 Les Voyages lointains des écrivains polonais $\left(\mathrm{XX}^{\mathrm{e}}-\mathrm{XXI}^{\mathrm{e}}\right.$ siècles $)-\mathrm{n}^{\circ} 51$

essentiel est la satire tantôt empathique, tantôt distanciée et mordante (ayant pour cible des comportements politiques inappropriés, qu'ils émanent de milieux non tziganes, tziganes ou d'individus pour lesquels cette étiquette est surtout un moyen commode pour tirer profit des aides proposées par les pouvoirs publics). Le second ouvrage, quant à lui, se rapporte à des communautés et des espaces considérés comme « lointains », en l'occurrence, le Canada et ses forêts peuplées d'Indiens, que l'auteur explora dès les années 1930. Kanada pachnaca żywica [Le Canada qui sent bon la résine] d'Arkady Fiedler se présente sous la forme d'une trilogie ${ }^{5}$ regroupant des récits de voyage et des reportages littéraires publiés pour la première fois en 1935 et réédités à de multiples reprises par la suite ${ }^{6}$. Cet ouvrage a été traduit en neuf langues étrangères, mais pas en français ${ }^{7}$. Cette fois-ci, le « je » scripteur est présent de manière explicite, et ce sont ses activités qui constituent le fil permettant d'enfiler les épisodes successifs.

Les deux écrivains, chacun à sa manière, deviennent des exilés, en ce sens qu'ils se déplacent - qu'importe que chez eux ce soit délibéré (on pourra parler d'exil volontaire) - en dehors de leur groupe d'appartenance, d'où résulte une contestation des cadres de représentation de celui-ci. Tous deux, ils visent à créer d'autres cadres, des cadres autres, à la faveur d'une expérience commune avec l'autre/(l')étranger, de fréquentation directe de cet autre. Dans les deux cas, la découverte de l'altérité progresse le long d'un axe qui est celui de l'exil par rapport à sa communauté d'appartenance et ses cadres de représentation, voire ses mécanismes d'exclusion. Notons toutefois que les visions stéréotypées sont réversibles et que les deux écrivains ont conscience que les Indiens, d'un côté, et les Tziganes, de l'autre, ont élaboré des concepts qui leur servent à se couper de «leurs » étrangers : les «gadjé » et les «visages pâles ». Chez les deux écrivains, la fréquentation de l'autre suscite une représentation de celui-ci en tant qu'être multiple, à bien des égards hybride, chez qui la pluralité s'avère constitutive aussi bien à travers l'histoire

5. L'exploration de l'espace canadien est scandée en trois étapes, à l'origine d'une division de l'ouvrage en trois grandes parties intitulées respectivement : « Il commence à sentir bon » $[$ Zaczyna pachnieć $]$, « L'excursion » $[$ Wyprawa $]$ et « Un paradis pour les chasseurs » $[R a j$ myśliwski].

6. La première réédition du Canada qui sent bon la résine affranchie de la censure communiste et intégrant les ajouts apportés par l'auteur au fur et à mesure des rééditions successives encore censurées ne voit le jour qu'en 2009, soit - finalement - à peine quatre ans avant la publication de l'ouvrage de Milewski. FIEDLER, 2009.

7. En 1974 (dernières données disponibles consultées), les ouvrages de Fiedler avaient été traduits en 23 langues. Voir: https://www.polskieradio.pl/39/156/Artykul/ 988376, Arkady-Fiedler-Bylem-wielkim-marzycielem. 
À Suwałki en Pologne et au Canada. Tziganes, Indiens, métis et êtres hybrides. Du cliché de l'étranger à l'hybridité exilique constitutive de la condition humaine

Piotr Bilos

que dans l'instantanéité. D'autre part, cet autre révèle son potentiel de proximité au sein même de l'altérité. Né du franchissement des frontières, l'exil invite à déstabiliser celles dans lesquelles a tendance à s'enfermer l'espace natal, domestique et familier par rapport à un « étranger » dont ne sait pas grand-chose sinon qu'il s'oppose au «nous », il devient un moyen permettant d'échapper à la logique binaire et essentialiste adoptée par les collectivités vivant sur le mode de la séparation et du refus de coopérer. Dès lors, l'écrivain et, à sa suite, le lecteur autochtone qui reste le destinataire principal, projeté et «sous-jacent » de ses textes, sont amenés à relativiser la frontière séparant l'altérité du chez-soi et à s'interroger sur les limites de la stabilité ontologique des identités respectives. Ce que ces ouvrages découvrent, n'est-ce pas - au bout du compte - une forme d'égalité des hommes vis-à-vis d'une condition exilique intrinsèque et transfrontalière ?

Aussi bien chez Fiedler que chez Milewski, le projet littéraire, qui s'origine dans la décision prise par le sujet-scripteur de s'extraire en dehors de sa communauté d'appartenance en vue de (ré)apprendre à vivre auprès d'étrangers (et dans une certaine mesure avec eux), devient le vecteur de la mise en forme de cette dimension essentielle.

Notre étude va donc privilégier une problématique à deux volets. Tout d'abord, nous partirons du constat que les deux ouvrages développent leurs architectures respectives à partir de la figure centrale incarnée dans le désir d'approcher un groupe de dominés sur le plan symbolique, matériel, social et historique. Sur ce plan, le regard des deux écrivains sur les autochtones et allogènes dominés s'avère étonnamment proche.

Toutefois, aussi bien les Tziganes que les Indiens ainsi que les autres groupes dominés et minoritaires n'incarnent pas uniquement une forme d'altérité et d'étrangeté ; leur existence plonge ses racines dans un mode de vie et des cultures jugés archaïques et démodés selon les normes de la société occidentale moderne. Se rapprocher d'eux signifie donc non seulement s'ouvrir à une altérité dominée, mais aussi, d'une certaine manière, faire marche arrière par rapport aux progrès de la civilisation. Ainsi, le sujet de la fréquentation des groupes de dominés mène directement à la dernière partie dédiée au questionnement sur les rapports nature/ progrès qui est l'autre grande problématique autour de laquelle les deux ouvrages gravitent. Faut-il en conclure que Fiedler et Milewski font l'apologie exclusive du retour à un mode de vie naturel et traditionnel qui serait une manière d'enrayer les dysfonctionnements et les désastres causés par des sociétés engagées sur la voie de la modernisation et du progrès technique ?

Mais il y a plus : puisque le « je » scripteur (ou l'auteur) a adopté le principe d'une sortie en dehors de l'espace originel d'appartenance, ne serait-ce que parce qu'il souhaite enrichir sa communauté d'une connaissance plus juste d'un étranger 
SLOVO

158 Les Voyages lointains des écrivains polonais $\left(\mathrm{XX}^{\mathrm{e}}-\mathrm{XXI}^{\mathrm{e}}\right.$ siècles $)-\mathrm{n}^{\circ} 51$

relégué jusque-là dans le brouillard, on comprend pourquoi les deux écrivains, par-delà les seuls Indiens ou Tziganes, accordent une importance capitale à ceux que l'on pourrait qualifier d'autres au second degré : des autres par rapport à ces premiers autres que sont les Tziganes et les Indiens eux-mêmes. Tout d'abord, les métis, ensuite toutes sortes d'êtres hybrides, ceux à qui eurent à essuyer l'accusation de renégats, de traîtres ou qui font figure d'excentriques, d'originaux. Bref, nous retrouvons ici la figure de l'outsider dont parle Saï, une composante essentielle, selon lui, du statut de l'intellectuel, outsider et exilé à la fois.

Plus fondamentalement, on en arrive à se demander si la découverte de l'hybridité ne contient pas une valeur opératoire universelle: elle affecte les communautés dominées aussi bien que les groupes dominants, quand bien même ces groupes pourraient refuser de le reconnaître.

C'est pourquoi la partie centrale de cette étude se focalise, par-delà la rencontre entre l'auteur et les minorités dominées du Canada telles qu'Indiens, métis et Français, sur un personnage intermédiaire et problématique lui-même : le guide-trappeur, ermite Stanisław, compagnon de route que s'est choisi Fiedler. Stanisław, c'est l'Européen, le blanc, le Polonais devenu canadien qui s'est réinventé à partir d'éléments qui, au départ, signifiaient l'étrangéité et la non-appartenance ; son expérience, exemplaire, condense ce que les deux écrivains ont chacun voulu explorer et faire ressortir, à savoir la tendance au métissage et à l'hybridation propre à chaque population en vertu de la pente exilique de l'expérience humaine. Ces individus hybrides incarnent de la façon la plus tangible la déstabilisation du bel ordonnancement des frontières préétablies entre le chez-soi et l'étranger. Or c'est (aussi) cette déstabilisation que souhaitent offrir en représentation à leurs lecteurs ces deux écrivains.

Notre étude ne sera donc pas comparative au sens où par comparaison il faudrait entendre une «étude contrastive », parce que ces deux ouvrages loin de s'opposer, de s'écarter l'un de l'autre, ont été « rapprochés» précisément parce qu'ils développent une optique commune en vertu de laquelle ils se complètent l'un l'autre.

\section{Une logique qui échappe au binarisme et marquée par l'hybridité}

Ce qui rapproche le plus efficacement l'ouvrage de Fiedler et celui de Milewski est que tous les deux privilégient l'optique d'un regard, à la fois compréhensif et distancié, qu'un observateur venu du dehors, appartenant lui-même à un groupe ethnique qui le rapproche des dominants, porte sur des communautés que le cours de l'histoire a eu pour effet d'aliéner, de déposséder d'un chez-soi auquel s'identifier et qui se retrouvent marginalisées face à des cultures dominantes, voire colonisatrices. 
À Suwałki en Pologne et au Canada. Tziganes, Indiens, métis et êtres hybrides. Du cliché de l'étranger à l'hybridité exilique constitutive de la condition humaine

Arrêtons-nous un instant sur cette notion de « chez-soi ». Quelqu'un pourrait en effet objecter que celle-ci n'est que difficilement transposable à des communautés perçues comme essentiellement nomades telles que les Indiens ou les Tziganes. C'est l'occasion de remarquer qu'un tel cliché représente une première manière d'opposer les «dominés » et/ou les « minoritaires » aux «dominants ». Ainsi, on sera sensible à la façon dont les deux auteurs abordent cette question avec l'intention de contester la rigidité de l'opposition ; à les lire, il devient évident que cette question de la sédentarité/sédentarisation a connu plusieurs phases, autrement dit, qu'elle revêt une dimension historique, et que de surcroît, quelle que soit l'époque considérée, aussi bien les allogènes que sont les Tziganes ou les autochtones minorisés que sont les Indiens, ces deux communautés ont toujours cultivé la notion de chez-soi et que leurs liens avec un mode de vie sédentaire sont beaucoup plus importants que ce qu'on pourrait penser.

Fiedler évoque cette question à propos des Iroquois et son rappel historique s'effectue en trois temps, au moins (Fiedler,1973, p. 74-75):

Budowali wielki osiedla $i$ zajmowali sie rolnictwem, lecz mimo to wojownicy spędzali większa częśc życia na koczowniczym rozbójnictwie.

Ils construisaient de grands villages et pratiquaient l'agriculture, mais malgré cela, leurs guerriers consacraient l'essentiel de leur temps au brigandage nomade.

[...] Nie zawsze byli silni i bitni. Zachowane tradycje i inne źródta pozwalaja przypuszczać, ze jeszcze na początku XVI wieku ojczyzna ich byty lasy na pótnoc od rzeki Św. Wawrzyńca. W pewnym okresie ulegli tam przemocy Algonkinów, którzy ich sobie podporządkowali, bądź też częściowo wyparli na potudnie.

Les Iroquois n'avaient pas toujours été puissants et combatifs. Les traditions conservées et d'autres sources permettent de supposer qu'au début du $\mathrm{XVI}^{\mathrm{e}}$ siècle encore, leur patrie se trouvait dans les forêts au nord du fleuve Saint-Laurent. À une certaine période de leur histoire, ils y succombèrent à la violence des Algonquins qui soit les soumirent, soit les contraignirent à se replier vers le sud.

Zjawisko pozornie niezrozumiale [...] a jednak prawdziwe. Nad Irokezami najzuchwalszymi i najbardziej meskimi Indianami panowaty kobiety. Matrony doszty do takiego znaczenia, ze one 
SLOVO

160

Les Voyages lointains des écrivains polonais $\left(\mathrm{XX}^{\mathrm{e}}-\mathrm{XXI}^{\mathrm{e}}\right.$ siècles $)-\mathrm{n}^{\circ} 51$

wptywaty na mianowanie wodzów. Do nich należata ziemia, ognisko, dom, wszelki sprzęt i wszelka zdobycz.

C'est un phénomène en apparence incompréhensible [...] et pourtant authentique: ce sont les femmes qui régnaient sur les Iroquois, les plus téméraires et les plus virils des Indiens. Les matrones avaient acquis une telle importance qu'elles influaient sur le choix des chefs. Ce sont elles qui possédaient les terres, les foyers, les maisons, tous les équipements et toutes les choses acquises en butin.

Ces quelques extraits se référant aux temps passés suffisent à montrer comment Fiedler problématise la question du rapport à la terre et au chez-soi, à une « patrie » lorsqu'il dépeint les Indiens. Ici, nous apprenons que le mode de vie sédentaire n'avait pas été étranger aux Iroquois et lorsque les hommes se trouvaient accaparés par la guerre, les femmes exerçaient le rôle de gardiennes du foyer. De plus, Fiedler ne manque pas d'insister sur la dépossession de leur « patrie » septentrionale dont cette tribu fit l'objet par le passé ; c'est un élément intéressant, d'une part parce qu'il indique qu'en effet, les territoires dont les Iroquois revendiquaient la possession étaient assimilés à un « chez-soi » (souvenons-nous de la citation d'Edward Saïd) situé dans les forêts au nord du fleuve Saint-Laurent, d'autre part, parce qu'il constitue une allusion discrète à l'histoire de la nation dont Fiedler est issu : la perte que les Polonais eurent à déplorer de leur propre État tout au long du XIX ${ }^{\mathrm{e}}$ siècle. C'est un élément de rapprochement et d'identité entre les Iroquois et ce « visage pâle » d'un type particulier puisqu'il est le représentant d'une nation victime de la colonisation.

Les extraits cités renvoyaient à l'histoire, mais l'essentiel du récit de Fiedler concerne le présent et s'attache à dépeindre les conditions de vie dans lesquelles les Indiens vivent actuellement (en 1935). De ce point de vue, on retiendra deux moments de l'ouvrage qui insistent sur leur mode de vie désormais sédentaire. Dans le premier passage, l'auteur nous emmène à Caughnawaga, une banlieue de Montréal située sur la rive droite du fleuve Saint-Laurent où sont installés les descendants d'anciens guerriers Iroquois; dès le premier voyage de Fiedler effectué dans les années 1930, ils vivent surtout des aides de l'État et des touristes auprès desquels ils se livrent à une forme indirecte de mendicité (recevant des gratifications en argent de la part des Américains surtout, très friands de la camelote qu'ils proposent et toujours prêts à récompenser d'une poignée de dollars la possibilité de se faire prendre en photo aux côtés d'un guerrier menaçant ou d'une belle Indienne). Comme on peut le voir à partir de cet exemple, la sédentarité n'entraîne pas une amélioration automatique des conditions de vie. Le second passage concerne une 
À Suwałki en Pologne et au Canada. Tziganes, Indiens, métis et êtres hybrides. Du cliché de l'étranger à l'hybridité exilique constitutive de la condition humaine

Piotr Bilos

autre communauté d'Indiens, les Cris (ou Eeyou) installés plus au nord, au bord du lac Obijuan/Obedjivan ${ }^{8}$ dans un village construit autour d'un poste ou comptoir de traite de la Hudson's Bay Company. Fiedler et son guide Stanisław sont ébahis de découvrir ce village imposant après avoir parcouru plusieurs jours d'affilée des territoires entièrement vierges de toute présence humaine. Les Indiens y utilisent sans réticences les bienfaits de la technologie, et Fiedler relève avec humour qu'à cet endroit, il semblerait qu'ils se déplacent uniquement en bateau à moteur alors que les blancs (c'est-à-dire lui-même et son compagnon) sont condamnés à faire usage de leurs bras pour ramer; cette observation humoristique est due au fait qu'avant d'accoster, leur canoë croise coup sur coup deux bateaux à moteur conduits par des Indiens (le premier conducteur, interrogé par Fiedler, a juste le temps de répondre qu'il en est le propriétaire avant de filer à toute allure), mais elle doit également être comprise comme faisant pendant à l'image de décadence sociale projetée par les Iroquois de Caughnawaga. On apprendra plus tard que ce village est approvisionné chaque jour par un hydravion et que seuls deux blancs y vivent, Mr. Frankland, l'agent de la HBC, et une enseignante, une Française, femme d'un certain âge alors déjà.

Chez Milewski, la question de la sédentarité et du rapport au chez-soi, semble, au premier abord, plus discrète. Il est vrai qu'il y a des raisons objectives qui permettent d'associer les Tziganes, bien plus fermement encore que les Indiens, au nomadisme. À cet égard, le titre même (certes polysémique) Je doute que vous parveniez à nous suivre s'avère éloquent et on remarquera que le recueil contient des passages faisant l'apologie de la vie nomade et permettant de justifier celle-ci (Milewski, 2013, p. 136):

Gadże nigdy nas nie lubili. Bo jak można lubić tych - powiadaja co wszędzie zachowuja sie jak u siebie? A przecież my jak ptaki. Nie takie, co w klatce śpiewaja na rozkaz, na zadanie wyuczona melodie, piosenke. Nie jak gotębie - gtupie ptaszyska, co bez wytchnienia gnaja tam, gdzie im kazali, ale jak te ptaki, co same sobie trase lotu wyznaczaja, przelatuja z drzewa na drzewo, z lasu do lasu, a gdy groźna zima idzie, odlatują tam, gdzie cieplej i bezpiecznej.

8. Le nom de ce lac a connu des variations d'orthographe importantes. Fiedler utilise l'orthographe «Obidjuan » forme altérée d' «Obedjiwan » dont la transcription dans la langue atikamekv standardisée est: «Opitciwan ». Cette appellation provient des racines oba ou obe signifiant « détroit», et djwan correspondant à « courir ». Ainsi, elle peut être traduite par « courant du détroit ». 
SLOVO

162 Les Voyages lointains des écrivains polonais $\left(\mathrm{XX}^{\mathrm{e}}-\mathrm{XXI}^{\mathrm{e}}\right.$ siècles $)-\mathrm{n}^{\circ} 51$

Les Gadjé ne nous ont jamais aimés. «Comment aimer en effet ceux qui se comportent partout comme s'ils étaient chez eux ? », ont-ils coutume de dire. Pourtant, nous sommes semblables aux oiseaux. Pas à ceux qui, enfermés dans une cage, entonnent à la demande, une mélodie, une chanson apprise par cœur. Pas aux pigeons, ces oiseaux stupides qui courent à perdre haleine là où on leur ordonne d'aller, mais comme les oiseaux qui choisissent de leur plein gré leur trajectoire de vol, vont d'un arbre à l'autre, d'une forêt à l'autre et, à l'approche d'un hiver vigoureux, s'envolent là où il fait plus chaud et où ils seront plus en sécurité.

Néanmoins, on remarquera tout d'abord que Milewski, sur un mode qui est à la fois poétique et philosophique, assouplit l'opposition entre itinérance et sédentarité : «La route avait fini par devenir leurs racines ${ }^{9}$ » (Milewski, 2013, p. 133).

De même, Milewski, qui connaît parfaitement l'histoire des Tziganes polonais, garde en souvenir le décret de sédentarisation imposé en 1964 par le pouvoir communiste, mais aussi les différentes politiques entreprises depuis 1989 visant à la fois à promouvoir la sauvegarde de l'identité, de la langue et de la culture des Roms et leur insertion dans la société polonaise (voir note 18). Mais il y a plus. On peut interpréter le recueil Je doute que vous parveniez à nous suivre comme entièrement sous-tendu par la recherche, ayant un caractère à la fois drôle et mélancolique, d'un chez-soi. Les anecdotes et les histoires rapportées, adaptées et transformées par Milewski traitent toutes de l'exil, de la chaleur (parfois perdue) du foyer, des tentatives de rétablir les liens perdus avec la communauté, voire la communauté elle-même. Le récit «Bonne nuit les enfants » fait allusion à l'époque reculée et enveloppée d'un brouillard que les historiens s'efforcent encore aujourd'hui de dissiper, celle ayant précédé le départ d'Inde ; elle était marquée par la sédentarité (Milewski, 2013, p. 134):

Szczęśliwie im się żyto $w$ tych Indiach naszych kochanych. W zgodzie $z$ sąsiadami, uprawiając roślinki, hodując zwierzęta, ptasiego mleka tylko im brakowato. A może nawet $i$ nie. Gdyby byli zmęczeni, siadali sobie $w$ potudnie pod palmami, chtodzac nogi $w$ morzu, $w$ oceanie wielkim i popijali mleko ze ściętych z krzaka kokosów. A 
À Suwałki en Pologne et au Canada. Tziganes, Indiens, métis et êtres hybrides. Du cliché de l'étranger à l'hybridité exilique constitutive de la condition humaine

Piotr Bilos

zagryzali ananasem, brzoskwiniq soczysta rosnaca na wyciagnięcie ręki. Winogronami, chatwa i melonem. Pili palmowe wino, tańczyli i spiewali przy ogniskach. I śmiali się do gwiazd, co wesoto mrugaty do nich i przyjaźnie.

Żyto im się jak $w$ raju, aż nadeszty zte czasy. Dlaczego? Rożnie powiadaja.

Ils avaient vécu heureux dans cette Inde bien aimée. En harmonie avec leurs voisins, en cultivant des plantes, en élevant des animaux, il ne leur manquait que « les p'tits laits d'oiseaux », notre guimauve polonaise. Ou peut-être même pas. Quand ils étaient fatigués, ils s'asseyaient à midi sous les palmiers, en rafraîchissant leurs jambes dans la mer, dans un immense océan, et ils buvaient du lait de noix de coco cueillies dans des arbustes. Ce lait, ils l'accompagnaient d'un ananas, d'une pêche juteuse qui poussait là, à portée de main. De raisins, d'un morceau de halva et d'un melon. Ils buvaient du vin de palme, ils dansaient et chantaient autour d'un feu. Ils riaient en s'adressant aux étoiles qui leur clignaient de l'œil amicalement.

Ils vécurent comme au paradis jusqu'à ce que le mauvais temps vienne. Pourquoi ? On raconte à ce sujet toutes sortes de choses.

Dans la péripétie finale, où sont évoquées les circonstances du génocide tzigane durant la Seconde Guerre mondiale, on apprend que ce monologue provient d'un récit oral qu'un grand-père adresse à ses petits-enfants, et, par le biais d'une lune parlant polonais dans le ciel au-dessus de Berlin, on en déduit que les personnages sont des Tziganes polonais en proie à la nostalgie de la Pologne, ce qu'avait déjà indiqué la référence citée ci-dessus aux «p'tits laits d'oiseaux », guimauve typique de ce pays. Plus généralement, tous ces récits nous montrent des Tziganes extrêmement sensibles aux liens familiaux et à l'idée de foyer, quant au premier récit « Konopnicki et les autres », il permet de rattacher les protagonistes à un lieu précis sur la carte de la Pologne auquel ils se montrent eux-mêmes très attachés, la ville de Suwałki qui revêt une importance particulière pour l'auteur puisque c'est là qu'il a codirigé de 1993 à 2009 une école tzigane.

Ainsi, les deux ouvrages font glisser les Tziganes et les Indiens en dehors de l'opposition sédentarité/nomadisme; c'est là une première façon de brouiller la frontière, par essence, binaire et dualiste, qui, dans une vision stéréotypée, oppose ces groupes de minorités dominées aux groupes dominants. De fait, la non-sédentarité a longtemps été utilisée comme une arme de propagande contre ces groupes. Il est important de noter que sur ce plan, l'approche des deux écrivains coïncide 
SLOVO

164 Les Voyages lointains des écrivains polonais $\left(\mathrm{XX}^{\mathrm{e}}-\mathrm{XXI}^{\mathrm{e}}\right.$ siècles $)-\mathrm{n}^{\circ} 51$

avec les recherches récentes menées sur ces populations. Jolanta Drużyńska, dans Ostatni tabor [Le dernier convoi de roulottes], rapporte sur ce point les propos d'un sculpteur et poète tzigane polonais célèbre, Karol Parno Gierliński :

- Ta nasza wędrówka to byta wielowiekowa tradycja - podkreśla Karol Parno Gierlinski. - Ja miatem $w$ rodzinie cyrkowców i czesto rozmawialiśmy na ten temat, bo życie cyrkowca też polega na ciagtym przemieszczaniu się. Nasze koczownictwo wynikato raczej $z$ konieczności, jakie nam w Europie stworzono. [...] Tam, gdzie byty warunki, gdzie mogliśmy się zatrzymać na dtużej, to myśmy zostawali na state. Nasze chęci do koczownictwa wzbudzaty raczej różne dekrety antycygańskie. Więc można powiedzieć, że to wędrowanie, koczownictwo byto juz od poczatku wymuszone. Do dziś mamy cale wioski czy miasteczka na Batkanach zamieszkane przez Romów, którzy już nie pamiętaja od którego pokolenia prowadzq osiadty tryb życia. Podobnie w Szwecji czy $w$ Anglii nigdy nie byto zakazu koczownictwa, a i tak większość prowadzi osiadty tryb życia. (Drużyńska, 2015, p. 48)

- Notre voyage s'inscrit dans une longue tradition, souligne Karol Parno Gierliński. Dans ma famille, il y avait des gens du cirque et nous évoquions souvent ce sujet, car la vie du cirque repose sur des déplacements constants. Notre nomadisme résulte plutôt de choses qui nous ont été imposées en Europe. [...] Là où les conditions s'y prêtaient, où nous pouvions rester plus longtemps, nous nous fixions. Ce sont les divers décrets anti-tziganes qui nous ont poussés à adopter une vie nomade. Ainsi, on peut dire que cette itinérance, ce nomadisme ont été depuis le début forcés. À ce jour, nous avons des villages ou des bourgades entières dans les Balkans habités par des Roms qui ne savent plus à partir de quelle génération ils mènent un mode de vie sédentaire. De même, en Suède et en Angleterre, le nomadisme n'a jamais été interdit et, pourtant, la majorité mène une vie sédentaire.

À cela s'ajoute que le peuple ancestral installé au nord de l'Inde au v siècle, qui avait franchi les montagnes afghanes et rejoint les Balkans, était en effet, à l'origine sédentaire. De même, les chercheurs qui redécouvrent l'extraordinaire diversité des Indiens d'Amérique du Nord et de leur mode de vie font remarquer que c'est l'arrivée du cheval importé par les Européens qui intensifia la pratique du nomadisme, car le cheval permettait de traquer le bison sur de plus longues 
À Suwałki en Pologne et au Canada. Tziganes, Indiens, métis et êtres hybrides. Du cliché de l'étranger à l'hybridité exilique constitutive de la condition humaine

Piotr Bilos

distances. Auparavant, il était fréquent que les Indiens fussent sédentaires ou simplement semi-nomades (tels les Anasazis du sud-ouest de l'Amérique du Nord $)^{10}$. De plus, différents groupes d'intérêts aux États-Unis et au Canada ainsi que les hommes politiques ont pendant longtemps contribué à répandre le mythe selon lequel les peuples indigènes d'Amérique du Nord avaient une compréhension limitée et assez floue des droits de propriété, en particulier de ceux relatifs à la terre. Les recherches récentes conduisent à réviser totalement ce jugement ${ }^{11}$.

\section{L'espace canadien : subdivisions et antagonismes}

Arkady Fiedler, attentif à scruter en vis-à-vis les péripéties historiques et les réalités contemporaines, est amené à montrer les limites d'une opposition simplement binaire et duale entre blancs et Indiens ou « Premières Nations » [First nations], terme sous lequel il arrive qu'ils soient aujourd'hui désignés de manière officielle. Plus exactement, son exploration méthodique de l'espace canadien tend à démontrer que l'opposition binaire et duale Indiens/hommes blancs ou « visages pâles » ne fonctionne qu'à un certain niveau de la réalité. Le récit fiedlerien, d'emblée, fait basculer le dualisme originel dans ce que l'on pourrait appeler la topographie d'une logique hybride. De fait, si son récit remonte la pente du temps, les références les plus lointaines restent indissociables des expéditions européennes ; abstraction faite du viking Erikson évoqué brièvement, le curseur du temps ne démarre qu'à la toute fin du $\mathrm{Xv}^{\mathrm{e}}$ siècle et l'expédition de Jean Cabot et ne s'affirme véritablement qu'au début du XVII ${ }^{\mathrm{e}}$ siècle avec l'établissement des premières, encore modestes, colonies. La conséquence de cette optique «post-colombienne » est que l'espace canadien donné à voir par Fiedler est façonné par une constellation de communautés nombreuses et très variées. Quant au groupe des dominés, il est lui aussi multiple et se subdivise en catégories parfois non seulement antagonistes, mais aussi contradictoires. Certes, il y a les Indiens, mais ceux-ci sont les descendants de tribus alliées tantôt avec les Français, tantôt avec les Anglais (et, de façon beaucoup plus discrète, les Hollandais) : les deux puissances colonisatrices majeures du passé. Font également partie de ce groupe les communautés francophones elles-mêmes étant donné que celles-ci - comme le rappelle Fiedler - ont perdu la bataille de la suprématie sur ces territoires. Très vite, une place importante revient aux métis, descendants de couples mixtes, indiens et européens. L'action conjointe, mêlée et

10. La bibliographie en anglais sur les Indiens sédentaires d'Amérique du Nord est assez riche. Voir un aperçu à la fin de cet article.

11. Voir Rodriguez, Galbraith \& Stiles, 2006. 
SLOVO

166 Les Voyages lointains des écrivains polonais $\left(\mathrm{Xx}^{\mathrm{e}}-\mathrm{XXI}^{\mathrm{e}}\right.$ siècles $)-\mathrm{n}^{\circ} 51$

conflictuelle, de toutes ces communautés, dessine ainsi un tableau riche et contrasté, en rupture avec la vision traditionnelle liée à l'idée de « Frontière » :

La Frontière est pour le Pionnier cette ligne mobile qui avance tous les jours vers l'Ouest vers l'Eldorado vers le Pacifique. Elle sépare les territoires de la nature sauvage, de l'Indien et de son mode de vie respectueux de la Terre mère de ceux du Pionnier, que ce dernier considère comme la civilisation. D’un côté, se situe la nature sauvage, de l'autre le peuple élu par Dieu.

La Frontière, c'est les terres vierges de l'innocence et du paradis perdu, l'image de la civilisation pastorale idyllique par opposition à l'univers urbain aliénant. C'est la caution de la pureté qui lave l'Amérique de ses péchés, du capitalisme, de l'industrialisation, de ses villes inhumaines. C'est la Terre où l'utopie se réalise, où l'homme blanc devient « le bon sauvage » dont rêvait le siècle des Lumières ${ }^{12}$.

À l'inverse de cette logique binaire, le «je » de l'explorateur polonais des années 1930 tire les conséquences engendrées à long terme de ce qu'on pourrait appeler une colonisation... hybride, car fondée sur des alliances croisées. Telle ou telle tribu indienne choisit de pactiser tantôt avec les Français, tantôt avec les Anglais, mais il arrive aussi qu'au sein d'une communauté globalement acquise à une alliance, pour des raisons liées à des circonstances locales, émerge une faction qui va se prononcer en faveur du rapprochement avec la puissance majoritairement considérée comme ennemie. Ainsi, explique Fiedler, les Iroquois étaient devenus les ennemis jurés des Français et s'étaient alliés avec les Anglais en vertu d'intérêts respectifs convergents : les Anglais voulaient surtout conquérir des terres tandis que les Français s'adonnaient en priorité à la traite des peaux de bêtes au poil soyeux et s'étaient alliés aux premières tribus rencontrées, Hurons et Algonquins. Or les Iroquois menaient la guerre à ces tribus. Pourtant... parmi les Iroquois, ceux qui étaient installés à Caughnawaga sur la rive droite du fleuve Saint-Laurent, à proximité de ce qui allait devenir Montréal, parce qu'eux s'étaient retrouvés en butte à l'hostilité des Anglais, préférèrent recevoir le baptême de la part des Français et se convertir au catholicisme! Ainsi que nous l'avons vu, ce sont leurs descendants que Fiedler rencontra à Caughnawaga, un faubourg de Montréal (Fiedler, 1973, p. 73-82). 
À Suwałki en Pologne et au Canada. Tziganes, Indiens, métis et êtres hybrides. Du cliché de l'étranger à l'hybridité exilique constitutive de la condition humaine

Piotr Bilos

La logique exploratrice du récit lui-même, telle qu'elle est développée dans le Canada qui sent bon la résine, conduit au constat que la colonisation eut pour effet d'engendrer des alliances à géométrie variable, hybrides, croisées, parfois contradictoires. En effet, si les alliances engagent des communautés entières, Fiedler rappelle qu'au sein de celles-ci, il n'était pas rare de voir se constituer des factions opérant des choix antithétiques à la majorité, entraînant des accords « contre-nature » (puisqu'en s'alliant avec l'ennemi, on risquait de combattre ceux des siens restés dans le camp opposé et donc d'être combattus par eux). Ainsi, le récit fiedlerien fait ressortir une triade Indiens, francophones, métis dont la structure déborde le stéréotype d'un affrontement binaire entre «visages pâles » et $\ll$ peaux rouges $\gg$.

\section{Les Tziganes milewskiens : une communauté proche et lointaine; lointaine et pourtant si proche}

Une différence majeure semble néanmoins séparer les deux ouvrages qui nous occupent. Arkady Fiedler s'écarte de son lieu propre à double titre : il quitte la Pologne et, en même temps, il quitte l'Europe. Chez Jacek Milewski, c'est la Pologne qui fournit le cadre des principaux faits et péripéties même si d'autres États voire continents sont évoqués. Toutefois, en dépit de l'arrivée ancienne des Tziganes sur le sol polonais (et d'une histoire des rapports polono-tziganes plutôt moins difficile que dans d'autres pays $)^{13}$, leur culture n'en demeure pas moins perçue comme

13. Comme le rappelle Jolanta Drużyńska, les Tziganes sont arrivés en Pologne au $\mathrm{XV}^{\mathrm{e}}$ siècle. Un siècle plus tard, des édits anti-Roms sont mis en place face à l'afflux de Tziganes fuyant les persécutions à l'Ouest de l'Europe. Le XVII siècle, catastrophique du point de vue de l'ancienne Respublica (qui doit faire face à des calamités sans précédents: soulèvements cosaques, invasions, guerres, etc.), s'avère paradoxalement favorable à leur acclimatation sur ces terres, puisque c'est l'époque où les rois de Pologne donnent leur accord à l'institutionnalisation de la fonction de dirigeants tziganes (chefs, anciens et roi) ainsi qu'à des formes d'autogestion (impôts, tribunaux). Au XviII ${ }^{\mathrm{e}}$ siècle se met en place l'académie Smorgonska (actuellement, Belarus) sous le patronage de Karol Stanisław Radziwiłł où des Tziganes dresseurs d'ours sont employés. En 1791, la Respublica accorde les droits civiques aux Tziganes : ils acquièrent le droit de se déplacer et de s'installer librement en Pologne. Au même moment, Marie-Thérèse d'Autriche, la Hollande et les États germaniques prennent la décision d'interdire le nomadisme et ordonnent la sédentarisation forcée des Tziganes. À la fin du XIX ${ }^{e}$ siècle, dans l'Allemagne du chancelier Bismarck, cette politique sera encore plus brutale. Quant à la Roumanie, ce n'est qu'en 1856 qu'elle met fin à l'esclavage des Tziganes. La II République (1918-1939) est marquée par les élections des rois tziganes qui attirent les foules et les autorités polonaises ainsi que des observateurs et des journalistes étrangers (plus précisément, le régime de la « Sanacja » mis en place par Piłsudski en 1926 jusqu’à sa mort en 1935, apporte un soutien ostensible aux Tziganes). Voir sur ce point Jerzy Ficowski 
SLOVO

168 Les Voyages lointains des écrivains polonais $\left(\mathrm{XX}^{\mathrm{e}}-\mathrm{XXI}^{\mathrm{e}}\right.$ siècles $)-\mathrm{n}^{\circ} 51$

étrangère par la culture autochtone dominante. À cela s'ajoute que Milewski ne se met qu'exceptionnellement en scène lui-même, et toujours de manière non-explicite (on a parfois l'impression que c'est lui que se cache derrière le « je », mais cela reste exceptionnel ; inversement, dans le onzième récit, on devine que le «il » du père de deux adolescentes croisées par une Tzigane dans un tram renvoie à lui ${ }^{14}$ ) et fait « directement » parler ses personnages. Ainsi, Milewski déstabilise lui aussi le clivage eux/nous en engendrant un effet de familiarité paradoxale venant briser le mur d'étrangéité qui sépare le lecteur « autochtone » des Tziganes.

Dans le premier récit, cet effet est encore renforcé par le fait qu'une icône du patriotisme insurrectionnel, linguistique et polonais (luttant contre la politique anti-polonaise des puissances partageantes et l'injustice sociale), est « interceptée » par la communauté tzigane : il s'agit de l'écrivaine et poétesse Maria Konopnicka (connue également pour ses contes pour enfants et nouvelles pour la jeunesse), liée à la ville de Suwałki, « petite patrie » de l'auteur et siège de l'école bilingue romani-polonais qu'il a dirigée pendant des années (voir notes 1 et 3 ). Ce récit nous immerge dans un échange oral entre les membres d'une communauté plurigénérationnelle. Le lecteur est ainsi introduit in medias res dans un milieu qu'il percevra, s'il n'est pas tzigane lui-même (et on suppose que ce sera majoritairement le cas, puisque le livre est écrit en polonais), comme décentré. Pourtant, en vertu des pouvoirs de la littérature, il peut entendre en temps réel ce qui s'y dit, et l'écart culturel mentionné à l'instant, du fait de la structure même d'un récit mimétique d'un échange verbal entre initiés, est comme en partie résorbé. L'auteur est invisible, et pourtant, c'est bien lui qui tire les ficelles. La structure et la dynamique de cet épisode vise non seulement à déconstruire le mur d'étrangéité séparant le lecteur « autochtone » des Tziganes, mais aussi, plus subtilement, à montrer que cette communauté, par-delà ses faiblesses, reste dépositaire de certaines valeurs interhumaines mises en péril par le progrès de la société moderne. Il semble que Milewski ait pris le parti de ne pas s'attaquer de front aux représentions stéréotypées que le lecteur pourrait avoir de la communauté tzigane (comment, en effet, convaincre ceux qui pensent déjà savoir et avoir raison ?) ; en revanche, il décide de

(1985, 1989) et les travaux plus récents (qui discutent certaines thèses de Ficowski) d'Alicja Gontarek (2016). Ensuite, ce sera l'Holocauste des Tziganes d'Europe perpétré par les Allemands nazis. En 1964, le pouvoir communiste introduit un décret ordonnant la sédentarisation. Depuis 1989, beaucoup a été fait par les pouvoirs publics de façon à améliorer la scolarisation et l'insertion professionnelle ainsi que l'intégration des Tziganes dans la société.

14. Voir le récit „Ciotka Farfurka jest zdziwiona” [La Tante Farfurka est frappée d'étonnement], dans Milewski, 2013, p. 174-184. 
À Suwałki en Pologne et au Canada. Tziganes, Indiens, métis et êtres hybrides. Du cliché de l'étranger à l'hybridité exilique constitutive de la condition humaine

Piotr Bilos

prendre son lecteur à revers, car les échanges verbaux dans la confidence desquels il place celui-ci évoluent dans le sens d'une peinture critique de la société polonaise, européenne, sédentaire à laquelle le lecteur « majoritaire »/ « gadjo » s'identifie.

Qu'est-ce qui dans ce récit donne l'impulsion à cette série d'échanges dialogués, apparemment tous axés autour du thème de la mort et des esprits ? Des individus ont «décapité » la statue de Maria Konopnicka se trouvant dans un parc du centre-ville de Suwałki. Selon les Tziganes, réunis pour une veillée vespérale dans une pièce éclairée par une simple lampe de plafond, les journalistes sont « comme des vautours », eux qui sont toujours à l'affût de la moindre information un peu sensationnelle. En dépit du caractère dramatique de ces événements, l'humour s'invite lui aussi par le biais de l'un des participants ignorant du fait qu'il s'agissait d'une statue et qui demande quelle a été l'attitude des ambulanciers. Mais alors que le conteur principal, Zoga, signale que les malfaiteurs (ils étaient deux) ont été repérés par la police, un autre participant, Kapralo, s'étonne qu'il puisse s'agir de «notre police» (Milewski, 2013, p.9), ce qui fait que la petite assemblée s'engouffre dans la brèche et entame une série de plaintes contre la mesquinerie des forces de l'ordre, qui préfèrent s'en prendre aux Tziganes et à leurs représentants les plus faibles pour des actes au bout du compte assez anodins alors qu' ils font preuve d'indulgence à l'égard d'affaires autrement plus graves commises par les « gadjé » (Milewski, 2013, ibid.).

Comme indiqué en note 3, Jacek Milewski émaille les échanges oraux d'énoncés en romani. Ce choix engendre un double effet venant renforcer la tendance générale à remettre en question l'irréversibilité des clivages. Si les emprunts au romani (systématiquement traduits dans les notes de bas de page) semblent renforcer le sentiment d'authenticité et d'étrangéité qui résultait déjà de l'identité culturelle des protagonistes, en revanche, les thèmes abordés au cours de la conversion témoignent de la participation de la communauté tzigane à la même société polonaise que le lecteur «gadjo »; de plus, elles illustrent sa connaissance de l'histoire et des phénomènes relatifs à la culture populaire contemporaine (films d'Hollywood et de Bollywood, chansons de variétés, tabloïds, chaînes de magasins, etc.). Un effet similaire est produit par l'adjectif possessif « notre » associé au substantif « police »: les Tziganes, quel que soit leur rapport à la nation polonaise, font partie de la société polonaise. L'étrangéité apparaît à partir de là relative, et le texte laisse entrevoir la possibilité d'un échange fondé sur des points de convergence et une compréhension commune de réalités partagées.

Mieux, l'épisode initial, le premier d'une suite de récits axés, comme nous l'avons dit, sur la mort et les esprits permet aux divers participants à la veillée d'exprimer leur profond attachement à des valeurs que le «progrès » de la société moderne met en péril. Le lecteur « éclairé » découvrira avec une certaine stupéfaction que 
SLOVO

170 Les Voyages lointains des écrivains polonais $\left(\mathrm{XX}^{\mathrm{e}}-\mathrm{XXI}^{\mathrm{e}}\right.$ siècles $)-\mathrm{n}^{\circ} 51$

la croyance en l'existence des esprits semble être une chose assez universellement partagée chez ces Tziganes (ceux-là en tout cas). Toutefois, ces récits de fantômes et de revenants sont autant d'apologues où l'on voit fleurir des valeurs interhumaines telles que par exemple l'affection filiale et ou conjugale (si forte qu'elle parvient à surmonter la mort de l'une des parties), la loyauté et l'empathie (évoquant la miséricorde en un sens chrétien). De même, le motif du suicide invite à s'interroger sur la fragilité consubstantielle à l'être humain en tant qu'individu séparé et à l'importance subséquente des liens avec les autres, voire avec une communauté. Or l'on sait à quel point la pensée des rapports entre l'individu conçu comme sujet libre et autonome et le collectif représente un enjeu problématique pour l'homme moderne. Ainsi, la communauté tzigane, par-delà ses faiblesses, s'avère dépositaire de certaines valeurs interhumaines susceptibles de revêtir un sens universel.

\section{L'hybriditépar-delà le dualisme et le (simple) métissage: Stanistaw et les autres}

À la différence du recueil de Milewski, l'ouvrage d'Arkady Fiedler met en scène le «je s scripteur dans le rôle d'un aventurier-voyageur ${ }^{15}$, lequel entreprend d'affronter les flots tumultueux du grand océan ${ }^{16}$ pour se rendre de l'autre côté, overseas, comme disent les Anglo-Saxons. Ici, le dépaysement est immédiat car c'est non seulement un autre pays, mais aussi un autre continent que va explorer le « je ».

Fiedler situe son récit entre, d'un côté, la nature - omniprésente et valorisée pour la façon dont elle est animée par le principe de préservation des divers équilibres que l'homme, pour sa part, en tant qu'il subit la pression de passions sociales irrationnelles, n'aura de cesse de transgresser - et le Ciel de l'autre côté. Toutefois, la sphère du divin reste beaucoup plus discrète, et l'on sent en outre que l'auteur analyse de façon distanciée les manifestations de la foi individuelle et l'organisation sociale mise en place autour des différentes églises et contrôlée par celles-ci (dans la plupart des cas, il s'agit d'églises catholiques, puisque la population francophone du

15. L'auteur, au moment de partir pour le Canada, peut se prévaloir de nombreuses expéditions, au Brésil, en Amazonie et dans la cordillère des Andes en particulier.

16. Le voyage s'effectue en effet par bateau à partir du port de Gdynia. En tout, Arkady Fiedler aura effectué durant sa vie six voyages au Canada. Le plus ancien, qui va donner naissance à cet ouvrage, a eu lieu en 1935. Les suivants se produisent en 1945, 1961, 1972, 1975. Le tout dernier eut lieu en 1980 alors que l'écrivain allait sur ses 86 ans. 
À Suwałki en Pologne et au Canada. Tziganes, Indiens, métis et êtres hybrides. Du cliché de l'étranger à l'hybridité exilique constitutive de la condition humaine

Piotr Bilos

Canada était alors très majoritairement catholique $\left.{ }^{17}\right)$. De cette peinture il résulte une image contrastée, car si les prêtres contribuent à instaurer un certain équilibre, ils entendent exercer en contrepartie un pouvoir sur leurs ouailles qu'ils tiennent enfermés, dans les cas extrêmes, « comme sous une cloche de verre en les tenant à l'écart des courants du progrès ${ }^{18} \gg$.

Toutefois, les questions liées à la spiritualité se manifestent également sur un plan non institutionnalisé. Cela se produit par l'entremise du personnage de Stanisław, un trappeur solitaire vivant dans une cabane située à quelques 150 kilomètres au nord d'Ottawa, perdue, comme on l'a vu, au milieu des forêts quelque part au bord de la rivière du Lièvre. Fiedler va s'installer dans cette cabane et construire avec Stanisław une relation fondée sur la confiance réciproque. C'est en compagnie de ce chasseur invétéré que l'écrivain-reporter effectue l'essentiel des expéditions servant de points d'appui à la structure de l'ouvrage.

Certes, Stanisław est un exilé polonais. Fiedler nous apprend qu'il est né dans le tronçon de l'ancienne Pologne annexé par la Prusse (à l'instar de Fiedler lui-même) et que c'est parce qu'il refusait d'être enrôlé, au moment du service militaire, dans les rangs de l'armée prussienne qu'il avait décidé de s'enfuir en Amérique où il exerça toutes sortes de petits métiers avant de rejoindre une communauté de Kachoubes installés à Otter Lake où il apprit l'art de la chasse. Abandonnant cette communauté, il partit vers l'ouest en direction de la rivière Lièvre et, dans une clairière bien abritée, il finit par construire cette cabane où il s'établit avec un chien pour seul compagnon. Certes, il lui arrivait d'accueillir des chasseurs venus de la lointaine Montréal, mais c'était uniquement pour l'automne et, à d'autres occasions, il pouvait aider des voisins installés sur les rives de la Lièvre.

Fiedler souligne que bien que Stanisław eût choisi cette vie de solitude, il était d'un naturel plutôt sociable. C'est aussi l'une des raisons pour lesquelles il aborde cette figure avec une sympathie sincère mêlée de distance, car Stanisław reste pour lui un mystère. Il se demande qu'elles ont pu être les raisons véritables l'ayant poussé à choisir cette vie (cet ermitage) en retrait de la société ? Certes, elle favorise une très forte proximité avec la nature tout en initiant Stanislaw à une spiritualité orientée,

17. Fiedler évoque en particulier les églises Saint-Joseph de Montréal et Sainte-Anne à Beaupré près de la ville Québec avec lesquelles est lié le destin de Stanisław car celui-ci a été guéri de ses maux d'estomac par le curé de Saint-Joseph et de son eczéma au doigt après avoir fait un pèlerinage à Sainte-Anne à Beaupré (FIEDLER, 1973, p. 27). Un autre lieu marqué par l'influence de l'église catholique est la paroisse de la cité du Val des bois (FIEDLER, 1973, p. 28-33).

18. Zamykali ich równocześnie jak pod koputa szklanq z dala od nurtów postępu, FIEDLER 1973, p. 180. 
SLOVO

172 Les Voyages lointains des écrivains polonais $\left(\mathrm{XX}^{\mathrm{e}}-\mathrm{XXI}^{\mathrm{e}}\right.$ siècles $)-\mathrm{n}^{\circ} 51$

elle, plus spécifiquement vers le ciel, y compris en un sens transcendant, mais pour l'écrivain-reporter, il est possible qu'elle cache autre chose. Ce personnage s'avère en effet fondamentalement problématique et, plus encore, il incarne la figure de l'hybridité, de l'ambivalence identitaire qui, il faut le dire, résonne fortissimo dès les premières pages du Canada qui sent bon la résine et constitue l'un des leitmotiv de cet ouvrage.

Certes, Stanisław, par-delà ses origines polonaises, incarne avant tout l'homme ayant opéré un retour à la nature (ses gestes sont lents et mesurés, il fabrique tous ses ustensiles lui-même et vit de chasse et de pêche). Ayant réglé son mode de vie sur celui de la nature canadienne, pour Fiedler, il joue le rôle d'un guide-initiateur à cette nature ; ainsi, l'excursion entreprise par ce dernier vers le nord du Canada à partir du hameau de Oskélanéo, en canoë le long de la rivière du même nom, n'advient que parce que le trappeur a accepté d'être de la partie et de prêter main forte à l'écrivain-reporter ${ }^{19}$.

Mais ce n'est pas tout. Stanisław n'est-il vraiment que ce trappeur-ermite ? Au cours d'une scène d'anagnorisis [reconnaissance], Stanisław finit par révéler l'origine véritable de sa vocation. Le lecteur apprend alors qu'il est un avatar du héros romantique ayant été entravé dans sa vocation à une vie sociale active et respectueuse de l'ordre établi à cause d'amours contrariées. Mais à la différence de Giaur, le héros byronien, ou de Jacek Soplica/Robak ${ }^{20}$, celui de Mickiewicz (Mickiewicz, Pan Tadeusz, 1992/1834), Stanisław semble avoir renoncé à la violence. En même temps, il n'a jamais totalement fait le deuil d'un amour de jeunesse malheureux (ayant occasionné un double parjure) et, finalement, c'est surtout à lui-même qu'il fait violence. Quant à sa retraite, elle apparaît comme une chape posée sur une souffrance refoulée. Pour l'écrivain-voyageur, il devient alors clair que les rituels religieux auxquels s'adonne Stanisław sont le résultat de la sublimation de cette souffrance et semblent offrir un exutoire au personnage.

19. Celle-ci débute avec la deuxième partie de l'ouvrage intitulée précisément : « Excursion », Fiedler, 1973, p. 89 : „Wyprawa”.

20. Jacek Soplica a renoncé à son identité et à toute vie privée (ainsi, il s'interdit d'entretenir des contacts avec son fils). Son rôle en tant qu'organisateur de l'opposition armée contre l'occupant se fait non seulement incognito mais aussi à partir d'une identité de substitution (censée annuler la précédente): Soplica était un «szlachcic» aux ambitions privées et sociales, Robak est un prêtre dont les ambitions restent communautaires et nationales. L'ouvrage de Mickiewicz tisse subtilement une antithèse Soplica/Robak tout en faisant ressortir ce paradoxe qu'il s'agit du même individu. C'est en cela que le personnage du récit de Fiedler se rapproche de celui de Mickiewicz, puisque dans un cas comme dans l'autre, l'élément déclencheur, ce sont des amours contrariées. 
À Suwałki en Pologne et au Canada. Tziganes, Indiens, métis et êtres hybrides. Du cliché de l'étranger à l'hybridité exilique constitutive de la condition humaine

Piotr Bilos

Fiedler, tout en respectant la profonde religiosité que dégage son hôte, n'en est pas dupe.

Que ce personnage acquière une importance majeure sur le plan de l'histoire est indéniable : guidant l'écrivain-reporter à travers la nature canadienne, surtout celle, sauvage, du nord, Stanislaw est une sorte d'alter ego, à la fois proche et très différent de l'auteur. Mais son rôle s'avère tout aussi crucial sur le plan de la structure de l'ouvrage. L'histoire de Stanisław fixe deux jalons essentiels de celle-ci, réunis ensemble par la chaîne des aventures de l'expédition commune en canoë vers le nord. Fiedler avait fixé le premier jalon très tôt, en faisant part au lecteur de ses doutes concernant le fait que cet homme « qui vivait dans la proximité la plus intense avec la nature, n'avait pourtant pas subi son empire le plus grand, à savoir l'instinct de reproduction ${ }^{21} \gg$. Cette question posée à Stanisław avait suscité une réponse évasive prétextant l'absence d'intérêt pour avoir des enfants. C'était une pierre d'attente pour le lecteur. Mais ce n'est que 140 pages plus loin que Stanisław, inspiré par les murmures des forêts et des lacs et se remémorant ses Kachoubes du Canada, finit par avouer que la jeune Polonaise qui avait accepté sa demande en mariage avait suivi un prêtre irlandais cherchant une gouvernante pour remplacer la sienne, et venait de convoler en justes noces. Officiellement, il n'était question que d'un travail provisoire, mais, non sans avoir bercé d'illusions son ancien amoureux, Agnieszka (ainsi se prénommait la jeune fille), ne revint plus jamais de son presbytère éloigné d'une centaine de kilomètres. C'est surtout ce second épisode, scène d'aveu et de reconnaissance, qui rapproche Stanislaw du Jacek Soplica/Robak du Pan Tadeusz de Mickiewicz.

Bien sûr, de la part de Fiedler, il s'agit d'un procédé de retardement et de suspens classique : le premier épisode suscite une attente qui dynamise la lecture. Mais, en même temps, il faut se demander si, là aussi, comme avec la posture érémitique de Stanisław, il n'y a pas autre chose. Qu' incarne en effet Stanisław dans la perspective du projet littéraire de Fiedler et de la modalité du regard qu'il privilégie ? Nous avons vu comment dans un premier temps, le dualisme eux/nous, avait été supplanté par la triade: Indiens (répartis en tribus et en factions antagonistes), hommes blancs, métis. Par le biais de personnages tels que Stanislaw, nous assistons à une nouvelle évolution. La catégorie « métis », renvoyant à des phénomènes naturels, généalogiques et génétiques, se trouve elle-même dépassée par des phénomènes culturels et existentiels : le métissage (entendu en un sens avant tout biologique) cède la place à la notion d'hybridité que l'on comprendra comme l'expression culturelle 
SLOVO

(et non seulement biologique) d'un choix personnel. Or Fiedler, en choisissant de se rapprocher au maximum du Canada et, par l'intermédiaire de Stanisław, de s'immerger, pour ainsi dire, dans son être intime (on notera la valeur symbolique de l'eau des fleuves, support principal de l'expédition en direction du nord) bouscule le clivage chez soi/étranger, et c'est pourquoi - logiquement - l'écrivain-reporter manifeste son intérêt pour l'hybridité. Celle-ci devient très vite un objet d'étude privilégié. Pour Stanisław, l'hybridité s'incarne dans une série de tensions qui, au sens fort du terme, habitent le personnage : tension entre des origines polonaises et une vie canadienne, entre la nature et le ciel (l'infini qu' il dévoile), entre une nature sociable et une vie d'ermite, entre la spiritualité et la violence des passions terrestres.

En fait, Fiedler nous mettait depuis longtemps sur la piste de l'hybridité comme principe explicatif susceptible de rendre compte de la nature composée de Stanisław: très vite, il brosse des portraits de personnages incarnant de la façon la plus tangible la déstabilisation du «bel ordonnancement des frontières préétablies entre le chez soi et l'étranger » signalée en introduction. En voici quelques exemples. Le premier, qui renvoie à la figure d'un grand explorateur, constructeur de forts, stratège et aventurier indomptable, bien que finalement malchanceux, ne marque encore qu'une première étape en direction de l'hybridité. René-Robert Cavelier, sieur de La Salle, né à Rouen à l'époque de Louis XIV, a rejoint le Canada à l'âge de vingt-trois ans où il se mit en devoir d'explorer les forêts le long des rives du Saint-Laurent et d'apprendre, comme le souligne Fiedler, les langues indiennes. Cavelier avait réussi à gagner la confiance de Louis XIV et à le convaincre non seulement de lui accorder une concession pour le commerce des peaux de castors, mais aussi de le laisser construire une chaîne de forts le long des Grands Lacs jusqu'au Mississipi, un fleuve dont il va devenir l'un des premiers explorateurs. Ses efforts vont l'amener à découvrir et à fonder la Louisiane en l'honneur de Louis XIV. Ayant à plusieurs reprises joué de malchance et subi les menées de rivaux jaloux, il finit par mourir assassiné par des membres de son propre équipage, le 16 mars 1687. Fiedler lui consacre le chapitre 7 de la première partie de son ouvrage (Fielder, 1973, p. 33-37). La deuxième figure apparaît elle d'emblée marquée du sceau de l'hybridité : il s'agit d'Archibald Belaney, surnommé «Grey Owl » ou «Wa-sha-quon-asin » qui veut dire la même chose en ojibvé. Né en 1888, son père était un Anglais d'origine écossaise, et sa mère, sœur cadette de la première épouse du père tôt décédée (le mariage avait été contracté lors d'un séjour aux États-Unis), était quant à elle anglaise. Archibald avait prétendu qu'elle était Indienne, originaire de la tribu des Apaches, mais lorsqu'il mourut en 1938, on découvrit qu'il avait trafiqué son autobiographie pour se construire une identité exclusivement amérindienne. Entretemps, Grey Owl avait épousé une métisse iroquoise de dix-neuf ans ayant impulsé la conversion de ce trappeur 
À Suwałki en Pologne et au Canada. Tziganes, Indiens, métis et êtres hybrides. Du cliché de l'étranger à l'hybridité exilique constitutive de la condition humaine

Piotr Bilos

invétéré en défenseur de la cause de la sauvegarde des castors : Anahaero, c'était le nom de la jeune femme, avait incité son mari à élever un couple de jeunes castors, et c'est à partir de cet épisode que celui-ci évolua vers l'écriture jusqu'à devenir un écrivain et conférencier très populaire, chantre de la préservation de la nature. Que Grey Owl se soit inventé une identité amérindienne, mettant tout en œuvre pour la rendre crédible comme lorsqu'il se présentait au public en habit traditionnel indien, ne fait que renforcer l'hybridité de ce personnage. Évoquons pour finir une troisième figure, celle d'Etienne Brûlé, fils d'un paysan de Champigny-sur-Marne, devenu un « Huron blanc » (Fiedler, 1973, p. 50). Cela a déjà été dit, les Français étaient moins intéressés par l'acquisition de terres pour s'y installer que par le commerce des peaux de castors. Ainsi, plus que les Anglais, ils avaient besoin de la collaboration des Indiens en tant que chasseurs et leur choix s'était tourné vers les Hurons et les Algonkins. À cet égard, on notera que Fiedler insiste sur le rôle des traducteurs :

Gdy twórca francuskiej kolonii kanadyjskiej, Samuel Champlain, w roku 1608 zaktadat podwaliny pod stolice Quebec, posiadat w stosunku do Indian jasny program: zdobyć przyjaźń i wspótprace Algonkinów [...] Champlain posytat więc do nich swych najzdolniejszych ziomków, których nazywanych interprètes - ttumaczami, pośrednikami.

Lorsque le fondateur de la colonie française au Canada, Samuel Champlain, jetait les bases en 1608 de ce qui allait devenir la capitale, Québec, il suivait une feuille de route claire à l'égard à des Indiens : conquérir [leur] amitié et [leur] collaboration [...]. [II] se mit donc à leur envoyer ses compatriotes les plus doués appelés « interprètes »-c'étaient des traducteurs, des intermédiaires.

Or les Hurons étaient installés à l'Ouest dans des zones encore non apprivoisées et Champlain prit la décision de placer à la tête de l'expédition Etienne Brûlé qui n'était alors qu'un jeune homme de dix-neuf ans mais qui, écrit Fiedler, « jurait déjà comme un charretier, buvait comme un évier et fricotait avec les Indiennes tel un lièvre en rut, et, allez savoir, ne croyait peut-être même pas en Dieu. Un voyou, un écervelé sans aucune morale ${ }^{22}$ !» (Fiedler, 1973, p. 51). Fiedler insiste sur l'apprentissage et la métamorphose du jeune homme : celui-ci réussit en effet à se concilier les faveurs des Hurons en lutte contre les Iroquois et en proie à la menace 
SLOVO

176 Les Voyages lointains des écrivains polonais $\left(\mathrm{Xx}^{\mathrm{e}}-\mathrm{XXI}^{\mathrm{e}}\right.$ siècles $)-\mathrm{n}^{\circ} 51$

d'une sédition intestine. Il devint surtout populaire auprès des jeunes, révoltés contre l'autoritarisme des sages de la tribu. Il saura tirer profit de ces tensions en adoptant une attitude politique lucide et mesurée : allié aux jeunes, il ménage la susceptibilité des sages en refusant le titre de chef lui-même, n'oubliant pas son but principal qui est de ramener la plus grande quantité possible de peaux de castors. Et lorsque ce sera chose faite et que Champlain lui offrira un gîte à la hauteur de ses exploits à Québec, Brûlé rétorque aussitôt qu'il préfère rentrer chez les Hurons. Fiedler attribue explicitement le motif de l'hybridité à ce personnage (Fiedler, 1973, p. 53):

Byt to niepospolity junak, nad wiek dojrzaty. Dziarski $i$ niepohamowany- zmystowy, rozwiazty i przebiegty- niespożyty, przedsiębiorczy, szalony, wesoty i braterski. [...] Byt przedziwnym skrzyżowaniem- jednakowo żaru i stali; polaczenie btahych kaprysów, wzniostych polotów i rzemiennej twardości.

C'était un cœur vaillant, étonnamment mûr pour son âge. Vif et indomptable ; sensuel, lascif et rusé ; entreprenant, fou, joyeux et fraternel. [...] Sa personnalité formait un exceptionnel mélange - elle était marquée à la fois par l'ardeur et l'acier, résultat d'une alliance entre des caprices insignifiants, des envolées sublimes et une dureté grossière.

Deux épisodes de son destin tel qu'il est rapporté par Fiedler, fait d'exploits triomphaux, mais dénoué par une fin tragique, achèvent de hisser ce personnage au rang du symbole de l'hybridité. Le premier intervient lorsque les Français alliés aux Hurons peinent à prendre d'assaut un village iroquois et qu'Etienne est fait prisonnier. Il supporte sans broncher les supplices physiques qui lui sont infligés, mais lorsque le chef Iroquois s'apprête à lui arracher son collier orné d'un crucifix, Brûlé fait mine d'être horrifié et a cette réplique de génie : «Si tu touches à cet objet, tu perdras la vie au cours de la prochaine bataille ${ }^{23}$. » Certes, on peut dire que c'est le crucifix qui a sauvé la vie d'Etienne, mais ce n'est que parce que son détenteur lui a fait endosser le rôle d'une amulette païenne ! Toutefois, la témérité tous azimuts dont fait preuve le « Huron blanc », y compris à l'égard de la religion, va finir par lui être fatale. Comme le rappelle Fiedler, Etienne avait attiré sur lui l'ire d'un groupe d'acteurs de plus en plus important dans un contexte marqué par la conversion au catholicisme des Indiens alliés aux Français contre les Iroquois : les 
À Suwałki en Pologne et au Canada. Tziganes, Indiens, métis et êtres hybrides. Du cliché de l'étranger à l'hybridité exilique constitutive de la condition humaine

Piotr Bilos

missionnaires. La posture « païenne » d'Etienne est vite devenue une épine au pied de ces missionnaires, au demeurant jaloux de sa popularité auprès des Indiens. Bien qu'Etienne lui-même s'efforçât d'avoir des relations correctes avec ces missionnaires, les Jésuites en particulier, ceux-ci, écrit Fiedler « voulaient le rabaisser en refusant de reconnaître ses services et ses qualités, préférant au contraire grossir ses péchés tout en en cherchant à miner sa popularité auprès des Indiens ${ }^{24} \gg$ (Fiedler, 1973, p. 56). Sur ces entrefaites, en 1629, les autorités françaises durent se retirer du Canada après une victoire militaire des Britanniques, et Etienne Brûlé, qui avait décidé de rester sur place, proposa ses peaux de castors aux nouveaux maîtres. Champlain, témoin de cette scène le jour même de son départ, apostropha Brûlé en l'accusant d'être un traître, ce à quoi Brûlé répondit qu'il était un Huron. Les missionnaires utilisèrent ce prétexte pour «neutraliser » cet impertinent lorsque trois années plus tard les Français reprirent la main au Canada : ils firent croire aux Hurons qu'il était urgent qu'ils se débarrassent de « ce dangereux traître » s'ils voulaient s'assurer de l'appui de Champlain. Les sages, eux aussi, firent entendre leur voix et un procès fut intenté par les Indiens à Brûlé qui s'acheva par une condamnation à mort, laquelle fut exécutée d'une manière assez peu conforme aux usages des missionnaires qui n'en demandaient sans doute pas tant : Etienne Brûlé fut offert en repas aux juges !

Chez Milewski, le motif de l'hybridité s'avère également important même s'il ne revêt pas la forme d'une série de portraits réalisés en pied comme chez Fiedler. Néanmoins, l'auteur contemporain n'a de cesse d'interroger les modalités d'échanges identitaires possibles d'une communauté à l'autre, ce qui ne l'empêche pas, en parallèle, de jeter la lumière sur les obstacles ou les malentendus qui rendent ces opérations extrêmement périlleuses et parfois vouées à l'échec. Nous nous contenterons d'évoquer deux moments. Un fait supplémentaire vient agrémenter le premier, occasion d'un rapprochement direct entre Pologne et Canada: nous apprenons en effet qu'un Polonais, Wiesław, responsable mais non-propriétaire d'une boulangerie, faisait régulièrement appel aux services de Parno, un Tzigane, pour étamer et blanchir les chaudrons de sa boulangerie. Mais cinq années avant sa retraite, Wiesław a été licencié « comme un chien » (Milewski, 2013, p. 24), ce qui l'a poussé à boire et alors sa femme l'a congédié à son tour. Lorsque Parno rentra du Canada (!), voyant la détresse de cet homme qui l'avait toujours traité d'une manière équitable, il décida de lui venir en aide et lui aménagea un petit logement dans la cave de sa maison ne lui demandant en échange que d'effectuer de petits travaux dans la propriété. Les paroles de Parno sont éloquentes eu égard au motif

24. Woczach Francuzów starali się umniejszać jego zastugi, odmawiać mu zalet, wyolbrzymiać jego grzechy, natomiast u Indian — podkopywać jego popularność. 
SLOVO

178 Les Voyages lointains des écrivains polonais $\left(\mathrm{XX}^{\mathrm{e}}-\mathrm{XXI}^{\mathrm{e}}\right.$ siècles $)-\mathrm{n}^{\circ} 51$

de l'hybridité : « [...] tous nous étions contents. Il était comme l'un des nôtres, et malgré cela, il semblait un peu différent, c'était de son propre gré25 » (Milewski, 2013 , p. 25). Wiesław meurt lui aussi d'une manière tragique : convié au réveillon de Noël de ses amis Tziganes, il s'effondre en larmes en pensant à son épouse qui l'a éconduit. Après le repas, il se décide à aller la rejoindre et, à son retour, il est complètement ivre. Lorsque tout le monde pense qu'il s'est enfin remis de son chagrin, on le trouve pendu dans sa chambre. Le second moment fait intervenir une jeune fille qui apprend que sa mère n'est pas sa mère biologique et que celle-ci, décédée prématurément, avait été épousée par son père policier malgré l'opposition catégorique de la famille tzigane de la jeune femme. Le père attendait que sa fille soit suffisamment mûre pour lui révéler la vérité sur son identité. L'hybridité s'incarne cette fois-ci dans la figure de l'ours en peluche de la jeune fille (Milewski, 2013, p. 170) :

- Twój mis ma cygańskie imię.

- Ryciorek?

- No, cygańsko-polskie/Ryćoro to niedźwiadek, miś. Mam tak go nazwala, gdy go kupita dla ciebie.

- Ton ours a un prénom tzigane.

- Ryciorek ?

- Oui, tzigano-polonais. Ryćoro veut dire un ourson, un petit ours.

Maman l'a nommé ainsi lorsqu'elle l'a acheté pour toi. Et c'est toi qui l'a prénommé Ryciorek.

Ryciorek, c'est en effet l'un des diminutifs polonais morphologiquement plausibles pour un terme tel que Rycoro.

Pour conclure cette partie disons que Stanislaw, Grey Owl, Wiesław mais aussi Parno et sa famille, le père de la jeune fille, son épouse tzigane morte prématurément et l'ours en peluche, tous ces personnages bousculent le clivage chez soi/étranger à l'instar de ce que réalisent le projet littéraire de Fiedler d'un côté et celui de Milewski de l'autre.

25. [...] Wszyscy byli zadowoleni. Byt jak swój, choć osobny trochę taki, ale to już na wtasne zyczenie. 
À Suwałki en Pologne et au Canada. Tziganes, Indiens, métis et êtres hybrides. Du cliché de l'étranger à l'hybridité exilique constitutive de la condition humaine

Piotr Bilos

\section{Conscience écologique, humaniste et progrès technique}

Ainsi, l'hybridité, même lorsqu'elle se solde par une issue tragique, exprime le désir de dépasser les antagonismes identitaires en vue de réaliser des figures destinales complexes, situées au-delà de la ligne de séparation des communautés. En même temps, si l'on se replace du point de vue des normes des groupes dominants, engagés dans le processus de colonisation et, plus largement, de contrôle technologique $\mathrm{du}$ monde, on est légitimement en droit de se demander si Milewski et, encore davantage, Fiedler ne font pas l'apologie de la bonne nature et d'un mode de vie traditionnel opposés aux pouvoirs corrupteurs de la société ?

Cette question doit être posée et nous allons voir que, selon nous, dans les deux cas, le dualisme nature/culture finit par être dépassé à travers l'éloge de l'instinct proprement humain qui pousse à inventer des outils capables, chez Fiedler, d'aller au-delà de ce qu'offre la nature et de dompter celle-ci (machines et artefacts permettent à l'homme de dominer les éléments), chez Milewski (ce qui a déjà été évoqué), des outils avant tout pédagogiques et éducatifs afin de permettre l'insertion des Tziganes au sein de la société polonaise, mais de telle sorte que cette insertion ne signifie pas l'extinction de leur identité culturelle, qu'au contraire, elle serve à la renforcer.

Indéniablement, l'ouvrage de Fiedler contient en filigrane une sorte de manifeste écologique. Celui-ci préserve toute sa force de nos jours d'autant que le lecteur de 2019 s'étonnera qu'on ait pu dès 1935 observer certains phénomènes. Par exemple, Fiedler décrit le problème de l'engorgement des routes par des voitures omniprésentes dans la vie des habitants de l'Amérique du Nord : outre leur destination première, elles servent sans doute à combler un vide intérieur chez eux. De même, on retiendra les chapitres sur les castors et les moineaux. Les premiers ont failli disparaître car ils sont devenus la cible de chasseurs bénéficiant de l'appui de l'État séduit par les avantages financiers liés au commerce de ces animaux (y compris à cause des sommes récoltées au titre de la délivrance des permis de chasse). Les seconds, en revanche, importés d'Angleterre, ont à ce point cru en nombre qu'ils ont fini par détruire les vergers, les champs et les vignobles. Or quel facteur, dans un cas comme dans l'autre, a entraîné ces déséquilibres ? Comme le souligne Fiedler, ce n'est pas simplement la loi économique du profit à tout prix, mais, plus insidieusement, les passions sociales des hommes. La peau de castor « met en valeur la beauté des femmes et renforce le statut des hommes ${ }^{26} \gg$ (Fiedler, 1973, p. 40), quant aux moineaux, les Américains désiraient impérativement eux aussi en avoir, 
SLOVO

180 Les Voyages lointains des écrivains polonais $\left(\mathrm{XX}^{\mathrm{e}}-\mathrm{XXI}^{\mathrm{e}}\right.$ siècles $)-\mathrm{n}^{0} 51$

car il s'agit d'un oiseau européen, the English sparrow. En effet, ils étaient alors en proie à « un complexe d'infériorité par rapport à la old fashionable England ${ }^{27}$ » (Fiedler, 1973, p. 68-72).

Est-ce à dire que l'ouvrage de Fiedler constitue une énième illustration du mythe du bon sauvage et de la bonne nature opposée aux pouvoirs corrupteurs de la société ? Rappelons que quelques années plus tard, en plein cœur des ténèbres de l'incorporation de la Pologne au III $^{\mathrm{e}}$ Reich et à l'URSS, le poète-essayiste Czesław Miłosz, dans ses Légendes de la modernité critiquerait la prétendue capacité de l'île déserte à protéger l'homme des vices de la civilisation [Miłosz, Legendy nowoczesności, 2009 (1945)]. Si l'on ne peut pas dire que Fiedler abonde entièrement dans ce sens, néanmoins, son ouvrage ne manquera pas d'apparaître comme extrêmement nuancé quant à l'appréciation de l'utopie de la nature bonne et sauvage.

En réalité, si dans Le Canada qui sent bon la résine, Fiedler estime qu'un dépassement du prétendu dualisme nature/culture est nécessaire et c'est pourquoi il fait l'éloge du métier d'ingénieur en tant qu'il exprime la capacité de l'homme à inventer des solutions artificielles qui sont susceptibles de mettre la nature au service de l'homme, bien sûr à condition que celui-ci sache, en parallèle, respecter celle-ci et que, sur un autre plan, il la considère non comme une servante mais une maîtresse, une muse. Un passage en particulier va développer ce motif et offrir ainsi un pendant dialectique à ceux qui sont dédiés à la célébration des attraits de la nature sauvage. Il s'agit du chapitre 11 de la İ̀re partie de l'ouvrage, mystérieusement intitulé «Pon-pon » (Fiedler, 1973, p. 58-63). Nous y découvrons un adolescent polonais de quatorze ans, Wincenty (ou éventuellement Witold) désigné par le diminutif Wicuś, qui s'adonne à la chasse au canard sauvage en compagnie de Fiedler lui-même. Wicuś est comme ensorcelé par les rituels engendrés par cette pratique. Pourtant, alors qu'il emprunte le chemin du retour en direction de la chaumière du trappeur Stanisław, ses pensées, elles, s'engagent soudain dans autres voies, inséparablement liées à la civilisation industrielle (Fiedler, 1973, p. 61-62) :

Pon-pon to przemyslna a chytra zabawka, made in Japan. To blaszany okręcik. W środku tkwit prosty, żelazny kociotek z dwiema rurkami, wychodzacymi z tylu do wody. Gdy rozgrzać kociotek podtożonym kawatkiem świeczki i czótenko puścić na wodę, stateczek nabierat życia $i$ wesoto sunąt naprzód, dudniac $z$ zanurzonych rurek: pong, pong, pong. (Fiedler, 1973, p. 60)

27. Kompleks niższości wobec old fashionable England. 
À Suwałki en Pologne et au Canada. Tziganes, Indiens, métis et êtres hybrides. Du cliché de l'étranger à l'hybridité exilique constitutive de la condition humaine

Piotr Bilos

Pon-pon, c'était un jouet intelligent et plein de ruse, made in Japan. Un voilier en acier. À l'intérieur, on trouvait une mini-bouilloire d'où sortaient, s'immergeant dans l'eau, deux petits tuyaux. Lorsqu'on chauffait la bouilloire à l'aide d'un morceau de bougie placé en dessous avant de lancer le petit bateau sur l'eau, celui-ci s'animait et filait joyeusement vers l'avant, en grondant de ses tuyaux immergés : pong, pong, pong.

Et le narrateur de conclure :

Mtody zapaleniec chciat na zasadzie pon-ponu zbudować na rzece Lièvre wielka tódź dla użytku Stanistawa. Lecz dotychczas nie dociekt tej zasady i od wielu dni szukat rozwiazania. [...] Wicuś miat umyst wynalazcy i będzie z niego kiedyś tegi inżynier, lecz na razie pon-pon ukrywat przed nim swoja tajemnice. (FIEDLER, 1973, p.60)

S'inspirant du pon-pon, le jeune passionné voulait construire une barque immense qui aurait permis à Stanisław de naviguer sur la rivière du Lièvre. Mais jusqu'à présent, il n'avait pas réussi à arracher le secret du bateau et, depuis plusieurs jours, il cherchait à percer ce mystère. [...] Wicuś avait l'instinct de l'inventeur et il deviendrait un jour un grand ingénieur, mais pour l'instant, le pon-pon refusait de lui livrer son secret.

Ce n'est pas un hasard si dans le même chapitre, l'adolescent et l'écrivain évoquent une île déserte et isolée au milieu d'un lac qu'ils viennent de repérer. Alors que Wicuś est prêt à s'embarquer sur le champ pour mener la vie d'un nouveau Robinson, Fiedler lui rétorque en riant «stop, tu oublies que cette île déserte, j’y rêvais il y a très longtemps », et « aujourd'hui, ces rêves se sont couverts de rouille et ont perdu leur fraîcheur. Le temps a dissipé l'ancien charme de l'île ${ }^{28}$. »

In extremis, les inclinations profondes de Wicuś le poussent du côté de l'attitude à la fois enchantée et désenchantée de l'écrivain :

Mtody zapaleniec chciat na zasadzie pon-ponu zbudować na rzece Lièvre wielka tódź dla użytku Stanistawa. Lecz dotychczas nie dociekt tej zasady i od wielu dni szukat rozwiąania. [...] Wicuś miat umyst

28. Tak, ale dzis te sny pokryty się troche rdza i wyptowiaty. Czas rozwiat dawny urok wyspy, FIEDLER, 1973, p. 61. 
SLOVO

182 Les Voyages lointains des écrivains polonais $\left(\mathrm{XX}^{\mathrm{e}}-\mathrm{XXI}^{\mathrm{e}}\right.$ siècles $)-\mathrm{n}^{0} 51$

wynalazcy i będzie z niego kiedyś tegi inżynier, lecz na razie pon-pon ukrywat przed nim swoją tajemnice.. (FIEDLER, 1973, p.60)

Wicuś était très lié à la nature environnante et lorsqu'il était à l'affût des canards, son cœur battait plus fort. Mais ensuite, au milieu de la forêt enchantée et en dépit des parfums champêtres et des cris des oiseaux whippoorwill - quelque chose de surprenant prenait corps : des rêves de constructions en acier, des rêves de soudage de métaux, les mesures liées à une invention en train de naître. Il s'avérait que le charme de ces mesures, de ces constructions d'acier était plus puissant que celui de la supposée toute-puissante forêt.

Ainsi, le recueil de Jacek Milewski et l'ouvrage d'Arkady Fiedler donnent au lecteur la possibilité de fréquenter des communautés ou des individus perçus comme allogènes ou étrangers et qui incarnent des modes de vie jugés archaïques, comme en témoigne la croyance aux esprits chez les Tziganes ou la proximité avec la nature sauvage chez Stanisław le trappeur et les Indiens Cris. Par ce biais, ils sont une invite à revenir à des valeurs menacées par le progrès de la civilisation. Est-ce à dire que ces deux ouvrages vanteraient exclusivement des modes de vie naturels, coupés de la modernité ? Que ce soit le lien établi entre la modernité et les progrès de l'éducation chez Milewski ou l'épisode avec Wicuś et ses rêves d'ingénieur nourris par l'artifice du pon-pon, ces éléments sont là pour montrer que les deux auteurs rejettent, chacun dans son domaine, toute espèce de nostalgie trop univoque, et qu' ils s'efforcent, au contraire, de penser l'articulation du naturel et du traditionnel avec un monde en plein devenir, engagé sur les rails du progrès technologique.

\section{Bibliographie}

\section{OEuvres}

Fiedler Arkady, 1973 [1935], Kanada pachnaca żywica [Le Canada qui sent bon la sève], Iskry, Varsovie, 275 p.

Fiedler Arkady, 2009 [1935], Kanada pachnaca żywica [Le Canada qui sent bon la sève], Bernardinum, Varsovie, $328 \mathrm{p}$.

Milewski Jacek, 2013, Chyba za nami nie traficie [Je doute que vous arriviez à nous suivre], WAB, Varsovie, $272 \mathrm{p}$. 
À Suwałki en Pologne et au Canada. Tziganes, Indiens, métis et êtres hybrides. Du cliché de

l'étranger à l'hybridité exilique constitutive de la condition humaine

\section{Àpropos des ouvrages étudiés et leurs auteurs}

Sur Arkady Fiedler

Kempinski Tomasz, 2016, „Egzotyczny świat Arkadego Fiedlera” in Argumenta Historica. Czasopismo Naukowo - Dydaktyczne, n³ pp. $42-59$.

Pleszkun-Olejniczakowa Elżbieta, 2005, „Reportaż: wokół pochodzenia, definicji i podziałów" in Acta Universitatis Lodziensis. Folia Litteraria Polonica, $\mathrm{n}^{\circ} 7 / 2$, pp. 3-27, https://urlz.fr/b8s $\mathrm{H}^{29}$.

SIKora Agnieszka, 2016, „Kanada pachnąca żywicą?” in Gazeta Uniwersytecka UŚ, Katowice, http://gazeta.us.edu.pl/node/226721.

Sur Jacek Milewski

WróblewsKA Natalia, 13/10/2013, „Chyba za nami nie traficie”, recension de l'ouvrage in Polskie Radio Biatystok.

GARAPICH Michał P., 25/10/2019, Chyba za nami nie traficie - migracje polskich Romów na przetomie XX $i$ XXI w. Perspektywa antropologiczna, séminaire inaugural d'un cycle axé sur les migrations, Uniwersytet Jagielloński, Cracovie.

Kowalska Dorota, 2016, Wspótczesne koncepcje edukacji etnicznej dzieci romskich w teorii i w praktyce in Kowalska Dorota \& Urszula Strzelczyk-Raduli (eds.), «Ważne sprawy naszych uczniów », Opole, 171 p, http://pedagogika.wszia. opole.pl/ebook/uczniowie2014.pdf.

„Kwartalnik Romski”, 2013 in Czerwiec Sierpień, n¹1/3, http://www. romowieradom.pl/psd/kwartalnik_romski_11_20133.pdf.

\section{Ouvrages secondaires cités}

BiŁos Piotr, 2012, Exil et modernité, vers une littérature à l'échelle du monde: Gombrowicz, Herling, Milosz, Classiques Garnier, Paris, 402 p.

29. Tous les articles ou ouvrages disponibles en ligne consultés le 20/11/2019. 
SLOVO

184 Les Voyages lointains des écrivains polonais $\left(\mathrm{XX}^{\mathrm{e}}-\mathrm{XXI}^{\mathrm{e}}\right.$ siècles $)-\mathrm{n}^{0} 51$

Mickiewicz Adam, 1992 [1834], Pan Tadeusz [Messire Thaddée], trad. fr. par LEgRAs Roger, Éditions L'Âge d'Homme, 304 p.

MiŁosz Czesław, 2009 [1945], Legendy nowoczesności [Les légendes de la modernité], WL, Cracovie, $324 \mathrm{p}$.

SAÏD Edward W., 1996 [1994], Des intellectuels et du Pouvoir, Seuil, Paris, 144 p.

Sur les Indiens sédentaires d'Amérique du Nord

McCane Mallory, 1995, Lost Cities of the Ancient Southeast, University Press of Florida, Gainesville, 216 p.

Brody Jerry, 1993, Les Anasazis: les premiers Indiens du Sud-Ouest américain, traduit de l'anglais par SEYrès Hélène, Edisud, Aix-en-Provence, 237 p.

Pauketat Timothy, 1994, The Ascent of Chiefs: Cahokia and Mississippian Politics in Native North America, University of Alabama Press, 235 p.

Pauketat Timothy R., 2009, Cahokia, ancient America's Great City of the Mississippi, The Penguin Library of American Indian History, Londres, 208 p.

Sur les Tziganes en Pologne et dans la littérature

DruŻYŃSKa Jolanta, 2015, Ostatni tabor [Le dernier convoi de roulottes], Editions Rebis, Poznań, 240 p.

Ficowski Jerzy, 1965, Cyganie na polskich drogach. Wyd. 2 poprawione i rozszerzone. [Les Tziganes sur les routes polonaises, $2^{e}$ édition revue et corrigée], Wydawnictwo Literackie, Cracovie, 389 p.

Ficowski Jerzy, 1989, Cyganie w Polsce. Dzieje i obyczaje [Les Tziganes en Pologne. Histoire et mœurs], Interpress, Varsovie, 289 p.

Mróz Lech, 1992, Geneza Cyganów i ich kultury [La genèse des Tziganes et de leur culture], Fundacja « Pro futuro », Varsovie.

Sobieska Anna, 2015, Dzieci Hagar, Literackie wizerunki Romów/Cyganów, Studia imagologiczne [Les enfants de Hagar, Représentations des Roms/ Tziganes, Études imagologiques], Oficyna 21, Varsovie, 326 p. 
À Suwałki en Pologne et au Canada. Tziganes, Indiens, métis et êtres hybrides. Du cliché de l'étranger à l'hybridité exilique constitutive de la condition humaine

Piotr Bilos

\section{Revues}

Brun Jean-Paul, 2003, « Voyage au cœur de l'identité culturelle de l'Amérique. L'aventure du Pionnier sur la Frontière » in Chevalier Thévenin (dir.), Frontière, Presses universitaires de Franche-Comté, Besançon, p. 11-21.

Gontarek Alicja, 2016, „Królowie cygańscy II Rzeczypospolitej. Wokół dorobku Jerzego Ficowskiego na temat sprawy cygańskiej w okresie międzywojennym” [Les rois tziganes durant la II ${ }^{\mathrm{e}}$ République. Autour de l'œuvre de Jerzy Ficowski consacrée aux questions tziganes pendant la période de l'entre-deux-guerres] in Annales Universitatis Peadagogicae Cracoviensis. Studia Historicolitearaia, cahier 16/2016, p. 145-158, https://cejsh.icm.edu.pl/cejsh/element/bwmeta1. element.ojs-doi-10_24917_20811853_16_11co (consulté le 20/05/2019).

Milewski Jacek, „Dlaczego jest tak źle, skoro jest tak dobrze? Rozmowa z Jackiem Milewskim" [Pourquoi les choses les choses vont-elles mal, puisque tout semble aller pour le mieux ? Entretien avec Jacek Milewski] in DobIAsz Maja \& SoŁtan Katarzyna, 2014, Kultura polskich Romów-Poznajmy się!, Centrum Edukacji Obywatelskiej, Warszawa (Varsovie), https://ceo.org.pl/sites/default/ files/kultura_polskich_romow_publikacja_2014_0_0.pdf.

Rodriguez Carlos L., Galbraith Craig S. \& Stiles Curt H., 2006, „American Indian Collectivism: Past Myth, Present Reality" in PERC Reports, vol. 24, $\mathrm{n}^{\circ} 2$, traduction française : «Les Indiens d'Amérique étaient-ils collectivistes ? » in Contrepoints, 2013: https://www.contrepoints.org/2013/11/13/146017-lesindiens-damerique-etaient-ils-collectivistes (consulté le 20/05/2019).

Le Canada qui sent bon la résine (première édition en 1935) d'Arkady Fiedler mêle le reportage à une quête personnelle. Les 14 récits du recueil Je doute que vous arriviez à nous suivre publiés en 2013 par Jacek Milewski exploitent la fiction afin de dire des vérités sur la communauté tzigane polonaise. Les deux ouvrages font apparaître une optique commune justifiant qu'on les étudie côte à côte. Arkady Fiedler et Jacek Milewski se déportent en dehors de leur groupe d'appartenance vers des communautés, certes ancrées solidement dans leur culture, mais aussi, à bien des égards, dominées et marginalisées : les Indiens et les Tziganes. La découverte de l'autre et sa transposition en récit s' inscrit dans une double perspective exilique, car l'exil touche aussi bien le sujet-scripteur que son objet d'étude. On pense à la figure de l'intellectuel, outsider et exilé, chez Edward Saïd. Né du 
SLOVO

Les Voyages lointains des écrivains polonais $\left(\mathrm{XX}^{\mathrm{e}}-\mathrm{XXI}^{\mathrm{e}}\right.$ siècles $)-\mathrm{n}^{\circ} 51$

franchissement des frontières, l'exil invite à déstabiliser les cadres de représentation de l'espace natal, domestique et familier, à faire l'épreuve d'un « étranger » à propos duquel nous ne savions pas grand-chose sinon qu'il était opposé au « nous ». Ici et là, le projet littéraire suppose une préparation quasi-scientifique; la mise en place de conditions quasi-expérimentales aptes à briser les obstacles qui, en temps normal, obscurcissent, voire rendent impossible l'accès à l'étranger. Fiedler s'est abondamment documenté sur le Canada et son histoire; son itinérance, loin des principaux foyers urbains, se fait le vecteur d'une découverte du «Canada profond ». Jacek Milewski a commencé à fréquenter les Tziganes en tant qu'éducateur; il a appris leur langue et n'a cessé de les côtoyer de près. Fiedler et Milewski bousculent le dualisme entre dominants et dominés. L'opposition Occidentaux/Indiens cède la place à une palette contrastée : Anglais, Français, factions indiennes alliées à tel ou tel groupe de Blancs, métis, émigrés. Chez Milewski, il se produit en outre un effet de familiarité paradoxale, fissurant le mur d'étrangéité entre le lecteur «autochtone » et les Tziganes. De plus, par-delà les Indiens et les Tziganes, les deux auteurs se tournent vers d'autres autres. Non seulement les métis, mais aussi des êtres marqués par l'hybridité, des individus ayant conjoint plusieurs identités culturelles et dont l'histoire revêt une dimension tragique: Etienne Brûlé, Grey Owl, le personnage de Wieslaw chez Milewski. Le guide Stanislaw chez Fiedler incarne lui aussi ce prototype. Ces autres au carré tendent un miroir au sujet-scripteur placé lui-même en décalage par rapport à sa communauté d'origine. Le mode de vie de Tziganes et des Indiens présente un certain nombre de traits traditionnels; proche de la nature, il est aussi dépositaire de valeurs interhumaines mises en péril par le progrès de la société moderne. De là naît un questionnement sur la modernisation, ses conséquences néfastes tout autant que sa profonde utilité. Ainsi, les deux ouvrages sont susceptibles d'alimenter les recherches contemporaines menées selon une perspective écocritique.

Mots-clefs : Reportage, autrui, voyage, nature et culture, progrès technique, autochtones, allogènes, étrangers, étrangéité, minorités, dominants et dominés, écologie, écocritique, Indiens, Tziganes, Roms, Gitans, Canada, valeurs, axiologie, éducation, société, nomadisme et sédentarité, révolte, héros romantique.

Arkady Fiedler's Canada Smels like resin (first edition in 1935) mixes reporting with a personal quest. The 14 stories of I doubt that you will be able to follow us (the volume was published in 2013) by Jacek Milewski exploit fiction in order to tell truths about the Polish Gypsy community. The two works reveal a common perspective that justifies studying them side by side. Arkady Fiedler and Jacek Milewski move outside their home group to communities, certainly firmly rooted in their culture, but also, in many respects, dominated and marginalized: 
À Suwałki en Pologne et au Canada. Tziganes, Indiens, métis et êtres hybrides. Du cliché de l'étranger à l'hybridité exilique constitutive de la condition humaine

Piotr Bilos

Indians and Gypsies. The discovery of the other and its transposition into a narrative is part of a double exilic perspective, because exile affects both the subject-writer and his object of study. We think of the Edward Said's figure of the intellectual, both outsider and exile. Born of crossing of borders, exile invites to destabilize the frameworks that belong to the native, domestic and familiar realm, to experience "foreigner" about whom we did not know much except that he is opposed to "we." Here and there, the literary project presupposes a quasi-scientific preparation; the establishment of quasi-experimental conditions capable of breaking down obstacles which, in normal times, obscure or even make access to foreign countries impossible. Fiedler has extensively researched Canada and its history; his excursion, far from the main urban centers, becomes the vector of a discovery of "deep Canada." Jacek Milewski began to frequent Gypsies as an educator; he learned their language and kept getting to know them closely. Fiedler and Milewski subvert the dualism between dominant and dominated. The Western/Indian opposition gives way to a contrasting palette: English, French, Indian factions allied with such and such a group of whites, metis, emigrants. In Milewski's work, there is also an effect of paradoxical familiarity, cracking the wall of strangeness between the "native" reader and the Gypsies. In addition, beyond the Indians and the Gypsies, the two authors turn to "other" others. Not only metis, but also individuals marked by hybridity who have united several cultural identities and whose history has a tragic dimension: Etienne Brûlé, Gray Owl, the character of Wieslaw in Milewski. Fiedler's Stanislaw the guide also embodies this prototype. These exarcerbated others hold up a mirror to the subject-writer who is himself out of step with his community of origin. The lifestyle of Gypsies and Indians has a number of traditional features; close to nature, it is also the repository of interhuman values endangered by the progress of modern society. Hence a questioning about modernization, its harmful consequences as much as its profound utility. Thus, the two works are likely to feed contemporary research conducted from an ecocritical perspective.

Keywords: Reporting, others, travel, nature and culture, technical progress, indigenous, non-native, foreign, foreignness, minorities, dominant and dominated, ecology, ecocriticism, Indians, Gypsies, Roma, Canada, values, axiology, education, society, nomadism and sedentary lifestyle, revolt, romantic hero.

Kanada pachnąca żywicą Arkadego Fiedlera (wydana po raz pierwszy w 1935 r.) udanie wiąze reportaż z realizacjąpodmiotowej strategii literackiej. Chyba za nami nie traficie, utwór sktadający się z 14 opowiadań Jacka Milewskiego (wydany w 2013 r.) traktuje fikcje jako nośnik wypowiadania różnych prawd o spoteczności cygańskiej, zwtaszcza w Polsce. Oba utwory tączy wspólna perspektywa, co uzasadnia ich wspólne badanie. Specyfika projektu Arkadego Fiedlera i Jacka Milewskiego zasadza się na tym, że obaj przenosza się poza rodzinna grupe w strone wspólnot, wprawdzie 
SLOVO

188 Les Voyages lointains des écrivains polonais $\left(\mathrm{XX}^{\mathrm{e}}-\mathrm{XXI}^{\mathrm{e}}\right.$ siècles $)-\mathrm{n}^{0} 51$

mocno zakorzenionych we wtasnej kulturze, lecz także pod wieloma względami $z$ dominowanych i zmarginalizowanych: Indian i Cyganów. Odkrywanie Innego i jego transpozycja za pomoca narracji to procesy wpisujace sie $w$ „wygnańcza” perspektywe, ponieważ wygnanie odbija sie zarówno na sytuacji pisarza jak i przedmiocie jego badań. Przywodzi to na myśl postać intelektualisty, outsidera $i$ wygnańca $w$ rozumieniu Edwarda Saida. Będace owocem przekraczania granic, wygnanie to silny bodziec do destabilizacji ram reprezentacji wtaściwych dla przestrzeni rodzimej, do doświadczania „obcego”, o którym niewiele wiemy poza tym, że przeciwstawia się nam. Wobu przypadkach literacki projekt opiera się na quasi-naukowych przygotowaniach; ustanowieniu quasi-eksperymentalnych możliwości pokonywania przeszkód, które $w$ normalnych czasach zaciemniaja, a nawet uniemożliwiaja dostęp do Obcego. Fiedler intensywnie badat Kanade $i$ jej historie a jego marszruta, odbiegająca rychto od gtównych ośrodków miejskich, to wektor odkrycia "gtębokiej Kanady". Jacek Milewski począt obcować z Cyganami jako pedagog - nauczyt sie ich jezyka i odtad stale zacieśniat taczace go z nimi więzy. Fiedler i Milewski poddaja rewizji dualizm między dominujacymi a zdominowanymi. Opozycja pomiędzy cztowiekiem zachodnim a Indianami ustępuje miejsca mocno skontrastowanej palecie: Anglosasi, Francuzi, ale także frakcje Indian sprzymierzajace się z taka czy inna grupa biatych, metysi, asymilujacy się emigranci itd. U Milewskiego ponadto występuje efekt paradoksalnej bliskości przetamującej ściane obcości między "rodzimym” czytelnikiem a Cyganami. Co więcej, obaj Autorzy poza Indianami i Cyganami zwracaja sie do, by tak rzec, innych innych. Nie tylko do metysów, ale także istot naznaczonych hybrydyzacja, mianowicie do jednostek taczacych w sobie kilka tożsamości kulturowych i których los przybiera czestokroć wymiar tragiczny: Etienne Brûlé, Gray Owl, postać Wiestawa u Milewskiego. Przewodnik Stanistaw u Fiedlera również uosabia ten prototyp. Owi inni „do kwadratu” ukazuja lustro pisarzowi, który sam przecież wyszedt poza wtasna wspólnote pochodzenia. Styl życia Cyganów oraz Indian nosi wiele tradycyjnych znamion; blizszy naturze, jest także skarbnica wartości międzyludzkich, którym zagraża postęp wspótczesnego spoteczeństwa. Stąd pytanie o modernizację, zarówno jej szkodliwe konsekwencje jak i gtęboka użyteczność. Zatem oba utwory stanowia pożywke dla kogokolwiek interesuje ekokrytyczna perspektywa w badaniach nad kultura. 